University of Louisville

ThinkIR: The University of Louisville's Institutional Repository

Electronic Theses and Dissertations

$5-2015$

\title{
A study of the catalytic and chemical properties of palladium and palladium-silver monolayer protected clusters.
}

Luther Benjamin Hutcherson

University of Louisville

Follow this and additional works at: https://ir.library.louisville.edu/etd

Part of the Chemistry Commons

\section{Recommended Citation}

Hutcherson, Luther Benjamin, "A study of the catalytic and chemical properties of palladium and palladium-silver monolayer protected clusters." (2015). Electronic Theses and Dissertations. Paper 2151. https://doi.org/10.18297/etd/2151

This Master's Thesis is brought to you for free and open access by ThinkIR: The University of Louisville's Institutional Repository. It has been accepted for inclusion in Electronic Theses and Dissertations by an authorized administrator of ThinkIR: The University of Louisville's Institutional Repository. This title appears here courtesy of the author, who has retained all other copyrights. For more information, please contact thinkir@louisville.edu. 


\title{
A STUDY OF THE CATALYTIC AND CHEMICAL PROPERTIES OF PALLADIUM AND PALLADIUM-SILVER MONOLAYER PROTECTED CLUSTERS
}

\author{
By \\ Luther Benjamin Hutcherson \\ B.S., Georgetown College 2010
}

\begin{abstract}
A Thesis
Submitted to the Faculty of the

College of Arts and Sciences of the University of Louisville in Partial Fulfillment of the Requirements

for the Degree of
\end{abstract}

\author{
Master of Science \\ In Chemistry
}

Department of Chemistry

University of Louisville

Louisville, KY

May 2015 

A STUDY OF THE CATALYTIC AND CHEMICAL PROPERTIES OF PALLADIUM AND PALLADIUM-SILVER MONOLAYER PROTECTED CLUSTERS

By

Luther Benjamin Hutcherson

B.S., Georgetown College 2010

A Thesis Approved on

April 20, 2015

By the following Thesis Committee:

Thesis Director

Francis P. Zamborini

Richard Baldwin

David Fraley 


\section{DEDICATION}

This thesis is dedicated to my family, and in particular my parents Luke and Susan Hutcherson who have been a constant source of support and encouragement throughout my educational career. 


\section{ACKNOWLEDGEMENTS}

I first would like to acknowledge my research advisor, Professor Frank Zamborini. He provided a constant source of encouragement, ideas, and motivation to achieve the work herein. Dr. Zamborini was very generous and accommodating in accepting me into his research group during a difficult time in my graduate education, and for that I am very grateful. His knowledge of the subjects his students study is immense, and he often has a "gut" instinct about what will work and will not work which helps students, myself included, pursue directions in their research that will be productive. While he is always ready to provide suggestions and guidance on a research project, he expects his students to work independently and proactively-something which will be helpful all in their future careers as scientists. He truly exemplifies everything one would desire in a mentor.

I would also like to acknowledge my other committee members, Dr Richard Baldwin and Dr. David Fraley.

I have had the opportunity to work with Dr. Baldwin in his role as advisor to the Chemistry Graduate Student Organization and through taking his courses. He has always provided excellent, if sometimes poignant, advice for me in all aspects of my time spent at the University of Louisville.

Dr. Fraley served as my undergraduate advisor, and his motivation and encouragement was a large part of the reason why I chose to attend graduate school in Chemistry. He is incredibly enthusiastic about the subject he teaches, and one cannot help but also get excited by listening to him. I can't recall the number of times when he sat down with me, one on one, to explain a complex subject in terms that I could understand, often interspaced with a lesson in life. Many times, these discussions were outside the scope of the courses he was teaching, but were things in which I took a personal interest. Dr. Fraley not only allowed-but encouraged-me to "get my hands dirty" with department instruments and not only learn how to operate them properly and interpret date from them, but also learn maintenance and troubleshooting. Few undergraduates get such an opportunity, and the lessons I learned from him, both in chemistry and in life, have been invaluable.

Dr. Cecilia Yappert has been an inspiration to me in my time at the University of Louisville. Although not my advisor, she has never failed to assist me in answering questions about my research. Furthermore, I can't begin to count the number of "bad" days I've had through my graduate career where Dr. Yappert was willing to lend a sympathetic ear and provide advice and encouragement. She truly is a special person, and I am eternally grateful to her just for her presence in the department. Encountering her in the hallway never failed to brighten my day.

Dr. Michael Nantz is currently serving as the department chair, but was serving as director of graduate studies when I started in this department. He was my first contact with the department, and when I visited in person he made me feel welcome and enthusiastic about joining this program. He has been a constant source of encouragement, as well as not being afraid to give me a "kick in the pants" when he thought I needed it. 
I am grateful for the time I spent under the mentorship of Dr. Frederick Luzzio, where I learned a significant amount of organic synthesis that has been beneficial to me in my later work.

Dr. Bill Richmond, Steve Riley, and Ms. Rodica McCoy have provided a tremendous amount of instrumental support to me over the course of my graduate career. Dr. Richmond has assisted me not only with support, but also has become a friend due to our mutual interests. Steve has worked many "miracles" with regard to repairing instruments which we thought were unrepairable, and can always be counted on to source repair parts. Rodica trained me extensively in the use of the thermogravimetric analysis (TGA) instrument, and also provided constant encouragement for my work.

Undergraduate student Alex Renn was a tremendous help to me in much of the work discussed in Chapter 3, dealing with palladium-silver alloy nanoparticles. $\mathrm{He}$ performed many of the nanoparticle syntheses as well as catalysis experiments.

The late Lars "Tracy" Larson provided a tremendous amount of help to me in providing needed chemicals as well as providing constant advice and encouragement. Ms. Renu Kakar has additionally helped me in the stockroom.

I would like to acknowledge both former and current group members. I have enjoyed the company of and working with all of the members of my group in my time at $\mathrm{U}$ of L. I would, however, like to mention two group members specifically. The first is Dr. Monica Moreno, who trained me in most of the experimental techniques I used. Monica is a brilliant scientist, and a person with whom I remain in contact. Despite the fact that she is no longer at $\mathrm{U}$ of $\mathrm{L}$, she has continued to assist me with my research. In my time working with her, she also became a close friend. I would also like to specifically mention Stacy Allen, who has become a close friend and confidante in the year since she joined our research group.

Finally, I would like to thank the University of Louisville and the Department of Chemistry for providing support for me through both a department fellowship and GTA assignments in my time in the program. 


\title{
ABSTRACT \\ A STUDY OF THE CATALYTIC AND CHEMICAL PROPERTIES OF PALLADIUM AND PALLADIUM-SILVER MONOLAYER PROTECTED CLUSTERS
}

\author{
Luther B. Hutcherson
}

April 20, 2015

This thesis describes a study of the ligand chemistry of Pd Monolayer-Protected Clusters (MPCs) synthesized in the presence of octylamine. MPCs consists of a metal core surrounded by various organic stabilizers. Pd MPCs coated with alkanethiols, alkylamine, dendrimers, surfactants, and polymers have recently been explored for catalysis applications.Pd MPCs coated with alkylamines are more catalytically active than alkanethiols for hydrogentation/isomerization of allyl alcohol, but at shorter chain lengths are unstable in $\mathrm{H}_{2}$. We explored Pd MPCs synthesized in the presence of octylamines and discovered that the ligands attach to $\mathrm{Pd}$ as octanenitrile. This amine to nitrile conversion is catalyzed by the $\mathrm{Pd}$ and occurs during reduction of $\mathrm{PdCl}_{4}{ }^{2-}$ with sodium borohydride. When nitrile-stabilized Pd MPCs are exposed to $\mathrm{H}_{2}$ or exposed to alkanethiols during a ligand place-exchange reaction, the nitriles desorb from the Pd surface and partially convert to amide and carboxylic acid groups. When the nitrilecoated Pd MPCs are heated ortreated with $\mathrm{H}_{2}$ or place-exchange with alkanethiols without $\mathrm{O}_{2}$, the nitrile ligands desorb from the surface asnitriles. The catalytic properties of dodecanethiol-coated PdAg alloy MPCs prepared by co-reduction of $\mathrm{Pd}(\mathrm{II})$ and $\mathrm{Ag}(\mathrm{I})$ salts showed an $\sim 70 \%$ reduction in catalytic activity towards 
hydrogenation/isomerization of allyl alcohol with just $10 \% \mathrm{Ag}$ incorporated into the alloy. We expected that PdAg alloys prepared by synthesizing dodecanethiol-coated Ag MPCs first followed by galvanic exchange with $\mathrm{Pd}(\mathrm{II})$ would produce more catalytically active MPCs with the Pd on the surface, however, difficulty in the isolation of PdAg MPCs prepared this way led to inconclusive results. 
TABLE OF CONTENTS

ACKNOWLEDGMENTS

ABSTRACT

LIST OF FIGURES

PAGE

iv

vi

$\mathrm{X}$

1. INTRODUCTION

1.1 Purpose of studies 1

1.2 Monolayer Protected Clusters (MPCs) 2

1.3 Examination of the Interaction between Pd and Ligands 4

$\begin{array}{ll}\text { 1.4 Catalysis using MPCs } & 8\end{array}$

$\begin{array}{ll}1.5 \text { Galvanic Exchange } & 10\end{array}$

2. EXPLORING THE LIGAND CHEMISTRY OF Pd MONOLAYER-PROTECTED

CLUSTERS DURING SYNTHESIS IN THE PRESENCE OF OCTYLAMINE AND

SUBSEQUENT EXPOSURE TO HYDROGEN, HEAT, AND THIOL PLACE-

EXCHANGE REACTIONS

2.1 Introduction

2.2 Experimental

2.3 Results and Discussion

2.4 Conclusions 
3. COMPARISON OF THE CATALYTIC EFFICIENCY OF PALLADIUM-SILVER ALLOY MPCS PREPARED BY CO-REDUCTION AND GALVANIC EXCHANGE

3.1 Introduction 39

3.2 Experimental 39

3.3 Results and Discussion $\quad 43$

3.4 Conclusions $\quad 52$

4. SUMMARY AND FUTURE DIRECTIONS

4.1 Conclusions from ligand removal studies $\quad 55$

4.2 Future directions of ligand removal studies $\quad 55$

4.3 Conclusions from Pd-Ag alloy catalysis study $\quad 56$

4.4 Future Directions of Pd-Ag alloy catalysis study 57

REFERENCES $\quad 59$

CURRICULUM VITAE 63 


\section{LIST OF FIGURES}

$\begin{array}{lll}\text { FIGURE PAGE } & \text { PAT }\end{array}$

1.1. Illustration of Metal Monolayer-Protected Clusters. 3

2.1(A) GC-MS chromatogram of Pd MPCs synthesized in the presence of octylamine in $\mathrm{CDCl}_{3}$. (B) Mass Spectrum of observed octanenitrile peak. The $\mathrm{C} 8 \mathrm{NH} 2$ ligands converted into $\mathrm{C} 7 \mathrm{CN}$ at some point during the reaction.

2.2. Reaction monitoring of the synthesis of C7CN Pd MPCs after (A) addition of $\mathrm{C} 8 \mathrm{NH} 2$ to $\mathrm{PdCl}_{4}{ }^{2-}$ and $\mathrm{TOABr}$ in toluene and (B) after subsequent reduction with $\mathrm{NaBH}_{4}$ by GC-MS.

2.3. GC-MS chromatogram of $\mathrm{C} 8 \mathrm{NH} 2 \mathrm{Au}$ MPCs after heat treatment at

$100{ }^{\circ} \mathrm{C}$ for $3 \mathrm{~h}$.

2.4. Thermogravimetric Analysis of C7CN Pd MPCs (A) Before $\mathrm{H}_{2}$ and

(B) After $\mathrm{H}_{2}$ exposure for $4 \mathrm{~h}$. 
2.5. GC-MS chromatogram of the supernatant of C7CN Pd MPCs in $\mathrm{CH}_{2} \mathrm{Cl}_{2}$ after 24 treatment with $\mathrm{H}_{2}$.

2.6. NMR spectrum of the supernatant of a solution of $\mathrm{C} 7 \mathrm{CN} \mathrm{Pd}$ MPCs treated with $8 \mathrm{~mL} / \mathrm{min}$ of $\mathrm{H}_{2}$ gas for $3 \mathrm{~h}$.

2.7. Schematic of the formation of the nitrile on the Pd surface during the synthesis of Pd MPCs in the presence of octylamine.

2.8. GC-MS chromatogram of the supernatant of C7CN MPCs treated with $\mathrm{H}_{2}$ in 28 the absence of $\mathrm{O}_{2}$.

2.9. Mechanism for the formation of amide from nitrile.

2.11. GC-MS chromatogram of supernatant after heating Pd MPCs at $100^{\circ} \mathrm{C}$ for $3 \mathrm{~h}$.

2.12. GC-MS chromatograms of supernatant of C7CN Pd MPCs after place-exchange with hexanethiol $(\mathrm{C} 6 \mathrm{SH})$ in the presence $(\mathrm{A})$ and absence (B) of $\mathrm{O}_{2}$. Pd MPCs were precipitated following place-exchange

2.13. Proposed mechanism of octanoic acid and octanamide formation during ligand exchange. 
3.1. (A) Experimental Set-up for examining hydrogen stability.

(B) Absorbance vs. Wavelength plot for Ag C12S MPCs in solution.

(C) Stability of Ag MPCs based on the absorbance as a function of time from 0-60 min under bubbling $\mathrm{H}_{2}$.

3.2. (A) $\mathrm{H}_{2}$ Stability of C12S Pd MPCs over time. (B) $\mathrm{H}_{2}$ Stability of

C12S Ag MPCs over time.

3.3. UV-Vis spectrum showing $\mathrm{H}_{2}$ stability of $\mathrm{C} 12 \mathrm{~S} \mathrm{Ag}: \mathrm{Pd}$ 50:50 MPCs prepared by (A) co-reduction and (B) galvanic exchange.

3.4. Gas chromatograms monitoring the hydrogenation/isomerization of allyl alcohol with $\mathrm{H}_{2}$ and the catalyst (A) C12S Pd MPCs and (B) C12S Pd:Ag 50:50 MPCs prepared by co-reduction.

3.5. (A) Percent conversion of allyl alcohol vs. time plot for (A) pure Pd C12S MPCs and (B) C12S 50:50 Pd:Ag MPCs prepared by co-reduction.

3.6. Plot illustrating percent conversion of allyl alcohol vs. Pd:Ag ratio for both co-reduction and galvanic exchange synthesis.

3.7. Gas chromatograms monitoring the hydrogenation/isomerization of allyl alcohol with $\mathrm{H}_{2}$ and the catalyst (A) C12S Pd MPCs and (B) C12S Pd:Ag 50:50 MPCs prepared by co-reduction. 


\section{CHAPTER 1}

\section{INTRODUCTION}

\subsection{PURPOSE OF STUDIES}

In recent years, there has been considerable interest in the synthesis and properties of metal nanoparticles (NPs). This interest is largely driven by their unique and tunable properties that are different from the bulk materials. ${ }^{1}$ A variety of transition metals have been used to synthesize metal NPs, including gold, ${ }^{2}$ nickel, ${ }^{3}$ iron (particularly iron oxide), ${ }^{4}$ platinum,${ }^{5}$ cobalt,${ }^{6}$ titanium,${ }^{7}$ ruthenium, ${ }^{8}$ rhodium, ${ }^{9}$ osmium, ${ }^{10}$ silver $^{11}$ and palladium. $^{12}$ In addition, particles consisting of alloys of noble metals, including platinum-palladium, ${ }^{13}$ platinum-gold, ${ }^{14}$ palladium-gold ${ }^{15}$ and palladium-silver ${ }^{16}$ have been prepared. Uses for metal NPs are widely varied. For example, metal NPs have been used for cancer treatment, ${ }^{17,18}$ sensing, ${ }^{19}$ and catalysis. ${ }^{20,21}$ Among catalysis applications specifically, metal NPs have been used to catalyze reactions as diverse as the FischerTropsch synthesis ${ }^{6}$, the Suzuki reaction, ${ }^{22}$ Heck reaction $^{23}$ and hydrogenation reactions. $^{24,12,25,26}$

For many years, our research group has worked on the synthesis and characterization of various nanomaterials. In particular, we have focused on gold, ${ }^{27,28,29,30,31,32,33,34,35}$ silver, ${ }^{36,37,38}$ palladium, ${ }^{39,40,41,42}$ and alloys of these. ${ }^{43,36,37,44,45,46}$ These nanoparticles have been synthesized for applications as diverse as 
sensing, ${ }^{42,28,30,32,40,37,45}$ catalysis, ${ }^{42,46,47}$ and other interesting uses. My primary research has focused on the characterization of the ligand chemistry of $\mathrm{Pd}$ and $\mathrm{Au}$ monolayerprotected clusters (MPCs) and altering the catalytic properties of PdAg alloy MPCs by the method of synthesis.

By examining the ligand chemistry of MPCs, we hoped to gain a better understanding of the chemistry which occurs at the Pd surface during the reactions which we examine in our lab. Through our work with galvanic exchange, we hoped to prepare MPCs which were both less expensive and more catalytically active.

\subsection{MONOLAYER-PROTECTED CLUSTERS (MPCs)}

A monolayer-protected cluster (MPC) consists of a metal core surrounded by stabilizing ligands (Figure 1.1). ${ }^{48}$ The purpose of the ligands is two-fold. First, bare metal nanoparticles have a tendency to aggregate into larger particles, especially when not in solution, unless they are protected by ligands or other type of stabilizer. ${ }^{40}$ Second, by careful selection of ligands, it becomes possible to tune the specific properties and function of the nanoparticles. ${ }^{46}$ It is thought that by using "bulky" ligands, access to the metal catalytic substrate by reactants in catalysis applications can be controlled, allowing the ability to tune the specific catalytic properties and stability of the MPCs. ${ }^{47}$

All nanoparticles synthesized in the course of this study were synthesized by using a variation of the two-phase process first described by Brust et al. in $1994 .{ }^{48}$ Briefly, a soluble salt of the desired metal is dissolved in in Nanopure (18M $\Omega-\mathrm{cm}$ resistivity) water. This metal salt is then transferred to toluene by the use of a surfactant. For all studies in this thesis, the surfactant used was tetra N-octyl ammonium bromide 


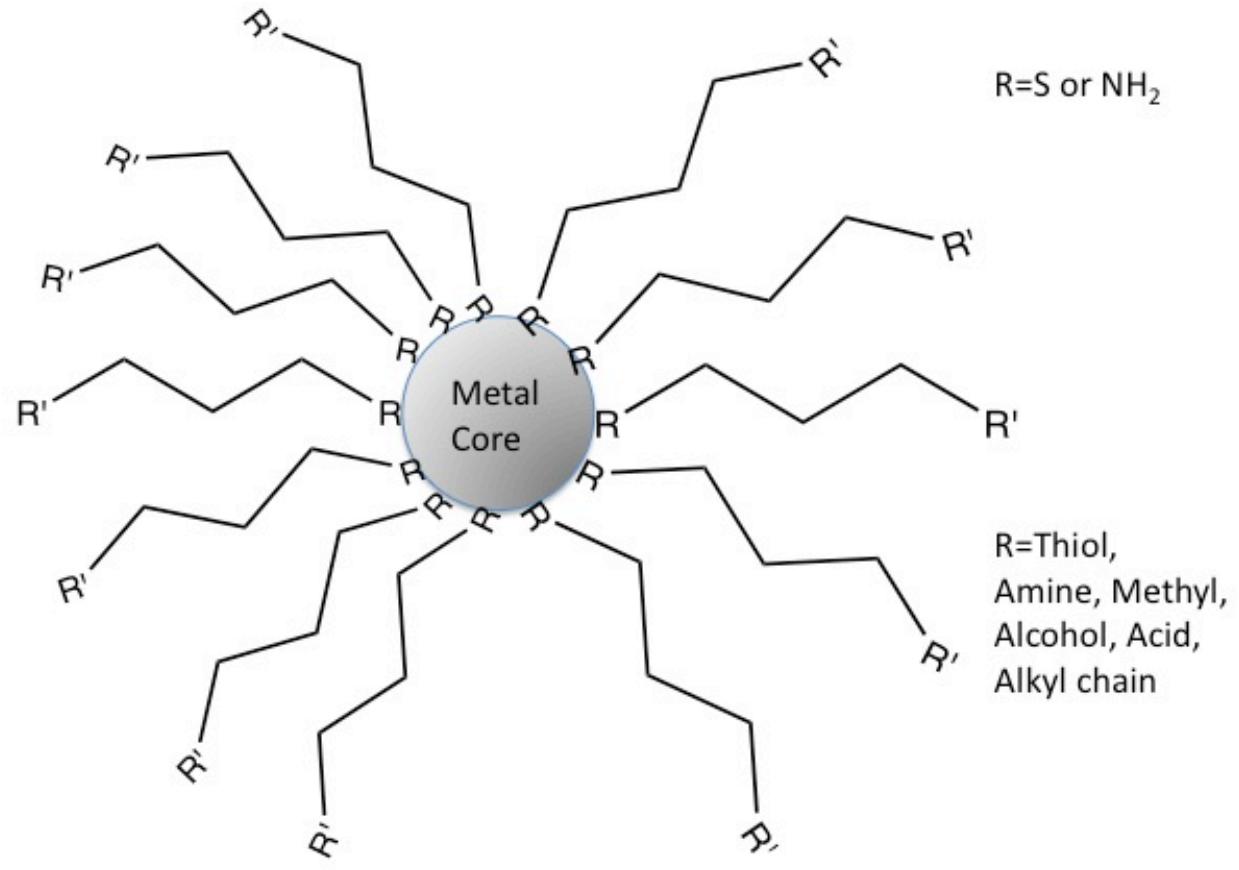

Figure 1.1. Illustration of Metal Monolayer-Protected Clusters. 
(TOABr). This results in phase transfer of the metal salt from water to toluene. The ligands are then added in the desired stoichiometric amount and the metal salt reduced insitu by sodium borohydride. The nanoparticles are then isolated and cleaned to remove excess ligands. In our experience these MPCs can be stored under ambient conditions for several days to several months depending on the nature of the metal and ligand.

The resulting MPCs can be readily re-dissolved in a variety of solvents, depending on the nature of the ligands. All MPCs examined through the course of this work were soluble in non-polar organic solvents. Toluene, benzene, dichloromethane, and chloroform were used for various experiments.

Through the course of these studies, it was found that when Pd MPCs are coated by amine ligands, the amine is reduced to a nitrile during the synthesis. Such a transformation is not observed when synthesizing Au MPCs coated by amine ligands. These findings are examined extensively in Chapter 2.

Applications for metal MPCs have been extensive and diverse. In addition to the catalysis work, our lab has also used MPCs for various chemiresistive sensing applications. Other groups have examined their electrochemical properties and use in nanoelectronics. $^{49}$

\subsection{EXAMINATION OF THE INTERACTION BETWEEN Pd AND LIGANDS}

Several different synthetic techniques have been used to prepare metal NPs, including electrochemical techniques, ${ }^{50,51}$ physical vapor deposition, ${ }^{52}$ thermal decomposition, ${ }^{14}$ chemical synthesis, ${ }^{53}$ biosynthesis techniques ${ }^{54}$ and microemulsion. ${ }^{6}$ Metal NPs have also 
been prepared using ionic liquids as solvents. ${ }^{52,55}$ Our primary focus is on MPCs prepared by solution based methods, using a chemical reducing agent and stabilizing ligands. ${ }^{53}$

Our group has recently focused on Pd MPCs. Due to the propensity for Pd to readily form hydrides, ${ }^{56} \mathrm{Pd}$ MPCs have been used for catalysis of hydrogenation reactions, ${ }^{57} \mathrm{H}_{2}$ storage, ${ }^{58,59}$ and $\mathrm{H}_{2}$ sensing. ${ }^{60}$ Pd MPCs have also been used for other types of catalysis including carbon-carbon coupling reactions such as the Suzuki reaction ${ }^{61}$ and Heck reaction. $^{62}$

A stabilizer is necessary to prevent the irreversible aggregation of Pd MPCs. A variety of techniques have been used to prevent aggregation, including solid and solution supports, such as titanium dioxide, ${ }^{63}$ carbon, ${ }^{64}$ silica, ${ }^{65}$ polymers ${ }^{66}$ such as polyvinylacetate ${ }^{67}$ or polyvinylchloride, ${ }^{68}$ biopolymers such as cellulose,${ }^{69}$ tin oxide, ${ }^{70}$ and dendrimers. ${ }^{71,72}$ Ligands such as isocyanide ${ }^{73}$ have also been used. Of particular interest to our group have been Pd MPCs with a self-assembled monolayer of alkanethiol or alkylamine stabilizing ligands. ${ }^{74}$ Metal MPCs coated with a self-assembled monolayer of organomercaptan ligands were first reported in 1994 by Brust et al. ${ }^{53}$ and consist of $\mathrm{Au}$ NPs coated with a monolayer of dodecanethiol ligands. In the time since that first publication, thiol-coated NPs with $\mathrm{Au}, \mathrm{Pt}$, and Pd cores have been prepared and used extensively, ${ }^{75-77}$ as well as thiol-stabilized NPs with Ag and AgPd alloy cores. ${ }^{16}$ Various groups have also used a similar synthesis, but with other types of ligand stabilizers, including alkylamines.

Ibanez ${ }^{60}$ et al. reported the preparation of solid state films of a variety of MPCs for $\mathrm{H}_{2}$ sensing applications. They examined both thiol and amine-coated Pd MPCs with ligands of a variety of lengths, including octylamine $\left(\mathrm{C} 8 \mathrm{NH}_{2}\right)$. Later, Moreno et al. ${ }^{47}$ used a 
variety of thiol and amine coated Pd and PdAg alloy MPCs to catalyze the hydrogenation and isomerization of allyl alcohol. By varying both the ligand composition as well as the $\mathrm{H}_{2}$ flow rate, Moreno was able to maximize the activity and control the selectivity of this reaction toward the hydrogenation or isomerization product.

In the work of Ibanez et al., ${ }^{43}$ they passed $\mathrm{H}_{2}$ over films of alkylamine-coated Pd MPCs while monitoring current through the film. The current increased dramatically in the presence of $\mathrm{H}_{2}$ initially and became stable after approximately 500 seconds for the octylamine-coated Pd MPCs. This indicated that the Pd MPCs irreversibly aggregated, forming a continuous metal film, upon exposure to $\mathrm{H}_{2}$. It was also noted that after $\mathrm{H}_{2}$ exposure, an IR absorption band at $2167 \mathrm{~cm}^{-1}$, corresponding to a nitrile stretch, disappeared from the film and all of the other bands of the original ligand decreased dramatically. This suggested that nitrile formation occurred during the synthesis and hydrogenation occurred after $\mathrm{H}_{2}$ exposure along with ligand desorption or other possible chemical transformations. Moreno et al. ${ }^{47}$ later studied the effect of $\mathrm{H}_{2}$ bubbling through a solution of various alkanethiol- and alkylamine-coated Pd MPCs, finding that Pd MPCs coated with alkanethiols (C6 or longer) and long chain (C16 or longer) alkylamines were stable in solutions in the presence of $\mathrm{H}_{2}$. Short chain octylamine-coated Pd MPCs aggregated and precipitated in solution within 5 minutes of $\mathrm{H}_{2}$ exposure. The formation of $\mathrm{PdH}_{\mathrm{x}}$ in the presence of $\mathrm{H}_{2}$ led to possible desorption of the alkylamine ligands and aggregation of the $\mathrm{PdH}_{\mathrm{x}}$ either because of lack of stabilizer interactions or crosslinked $\mathrm{PdH}_{\mathrm{x}}$ interactions. Moreno later monitored the solution species by NMR after $\mathrm{H}_{2}$ exposure and found several unidentifiable peaks that did not correspond to the original 
alkylamine ligand. These prior studies and preliminary observations in the lab provided the basis for the research presented here.

The chemistry of the stabilizing ligands both during exposure and after exposure to $\mathrm{H}_{2}$ is not well understood. It is known that atomic hydrogen tends to adsorb onto the surface of Group VIII transition metals to spontaneously form hydrides of these metals. ${ }^{78,79}$ The reaction of $\mathrm{Pd}$ with $\mathrm{H}_{2}$ occurs in two steps, with initial rapid and reversible adsorption of $\mathrm{H}$, with the $\mathrm{H}_{2}$ bond breaking to form Pd-H. This occurs on the palladium surface, followed by diffusion of atomic hydrogen into the metal lattice of the Pd. ${ }^{79}$ To effectively catalyze a hydrogenation reaction, the stabilizing ligands must allow $\mathrm{H}_{2}$ and other reactants to reach the Pd core, while still sufficiently preventing aggregation of the Pd. ${ }^{20}$ Longer chain lengths of stabilizing ligands tend to more effectively stabilize the Pd core, as do ligands which are more tightly bound to the Pd (i.e. thiols as compared to amines). Conversely, longer chain lengths of ligands and more tightly bound ligands tend to reduce accessibility to the Pd core, and reduce the catalytic activity of MPCs. ${ }^{16}$

In this thesis, we describe the ligand chemistry of the octylamine ligands after the synthesis of octylamine-coated Pd MPCs and the associated changes which occur upon exposure to $\mathrm{H}_{2}$ gas, heat, and ligand place-exchange with alkanethiols. The synthesis of alkanethiol-coated metal MPCs (particularly those with an Au core, but other metals as well), has been extensively studied. ${ }^{48,80,81,82,83,84,85,74,86,39,87,88}$ By contrast, very few studies have examined in detail the characterization of amine-coated MPCs. ${ }^{43,47,42,43}$

$\mathrm{H}_{2}$ exposure of the Pd MPCs is necessary to effect $\mathrm{H}_{2}$ sensing, hydrogenation reactions, and $\mathrm{H}_{2}$ storage. Thiol place-exchange reactions are necessary for functionalization and improving the stability of Pd MPCs. ${ }^{20}$ Heating is necessary for 
many catalysis reactions. To this point, the interaction of octylamine stabilizing ligands with the Pd core has not been well understood. For these reasons, we studied the ligand chemistry during and after synthesis, and after treatment with $\mathrm{H}_{2}$, heat and ligand placeexchange reactions.

\subsection{CATALYSIS USING MPCs}

The catalytic properties of Pd MPCs were studied by Moreno et al. previously in our research group. ${ }^{46}$ In particular, Moreno studied the catalytic activity of Pd MPCs on the isomerization and hydrogenation of allyl alcohol in the presence of $\mathrm{H}_{2}$. It was found that by changing the ligand on the Pd MPC, both the yield and selectivity of the reduction and isomerization products of this reaction could be tuned. Furthermore, changing the flow rate of the $\mathrm{H}_{2}$ through the reaction vessel also changed the selectivity of the reaction under study.

Although catalysis in our research group has focused on various alcohol hydrogenation and isomerization reactions, others have looked at the use of nanoparticles in catalysis of synthetic flavins, ${ }^{89}$ free radical polymerizations, ${ }^{90}$ peptide synthesis, ${ }^{91}$ improving the photocatalytic activity of $\mathrm{TiO}_{2} \mathrm{NPs},{ }^{92}$ diester and DNA hydrolysis, ${ }^{93}$ and CO oxidation. ${ }^{66}$

The use of Pd MPCs has been investigated extensively by our group, as well as others, for use in catalytic applications. The research in our group has focused on using Pd and PdAg alloy MPCs for catalysis of the hydrogenation and isomerization of allyl alcohol, which serves as a model reaction for potential catalytic applications. PdAg alloy MPCs prepared in our group previously have used co-reduction methods based on the 
Brust-type method previously discussed. Essentially, the Pd salt is dissolved in water and transferred to the organic phase using a surfactant. Another metal salt is then added, followed by the ligand, and both metals are reduced simultaneously using $\mathrm{NaBH}_{4}$.

Ibanez previously explored PdAg and PdAu alloy MPCs for hydrogen sensing applications, showing greater overall stability with PdAg compared to Pd MPCs alone. ${ }^{43}$ Moreno studied the use of various alkylamine coated PdAg MPCs for hydrogenation/isomerization of allyl alcohol, showing greater stability and activity compared to Pd MPCs coated with the same ligand. ${ }^{47}$ Others have looked at PdAu alloy MPCs. ${ }^{94}$ Alkylamine-coated MPCs resulted in the formation of both hydrogenation and isomerization products, while thiol-coated MPCs showed almost exclusive selectivity toward the isomerization product. The PdAg alloy is potentially important for catalysis applications involving $\mathrm{H}_{2}$ because $\mathrm{Ag}$ is much less expensive than $\mathrm{Pd}$ and $\mathrm{Ag}$ can adsorb large amounts of $\mathrm{H}$ as long as $\mathrm{Pd}$ is present to break the $\mathrm{H}-\mathrm{H}$ bond of $\mathrm{H}_{2}$. This makes it important to explore.

We previously synthesized PdAg alloy MPCs by co-reduction of Pd(II) and Ag(I) salts, which leads to a larger percentage of Ag on the surface according to Murray and co-workers. ${ }^{85}$ In order to obtain more Pd on the surface, we attempted here to synthesize Ag MPCs first and exchange the Ag on the surface with Pd by the galvanic replacement reaction, believing that this would improve catalytic activity while reducing the amount of Pd used (lower cost).

Galvanic exchange reactions-in this context-involve first preparing an MPC with an Ag metallic NP core. A Pd(II) salt is then added, and Pd atoms will exchange onto the surface and remove $\mathrm{Ag}$ atoms on the MPC surface (with one Pd atom replacing $2 \mathrm{Ag}$ 
atoms). It is thought that by doing this the catalytically active Pd atoms would be more accessible to the substrate than in the PdAg MPCs prepared by co-reduction.

The use of an alloy MPC for catalytic applications provides an obvious benefit in terms of cost. The much less expensive Ag can be used to provide the bulk of the MPC, with the more expensive but catalytically active Pd readily accessible on the surface.

In the research presented herein, the catalytic properties of PdAg alloy MPCs were examined with regard to hydrogenation of allyl alcohol. Catalytic examination of MPCs produced both by co-reduction and galvanic exchange were undertaken. This research is examined in detail in Chapter 3.

\subsection{GALVANIC EXCHANGE}

Traditionally, alloy NPs were prepared by the co-reduction of metal salts. Essentially, in a Brust-type synthesis, the metal salts of the two (or more) desired metals are combined before the addition of the surfactant, and the ligand then added. The metals are reduced simultaneously using sodium borohydride. ${ }^{46}$

In a galvanic exchange reaction, MPCs of a singular metal are first prepared. For the research shown in Chapter 3, Ag MPCs were first prepared. In a separate reaction, the salt of the secondary metal are then added, and through a galvanic exchange process the metal core of the MPC is "decorated" with atoms of the secondary metal. ${ }^{95,36,96}$ This type of process is ideal because the core of the MPC can be prepared using a less expensive metal (in this case $\mathrm{Ag}$ ), while the catalytically active metal (Pd) is deposited on the surface of the MPC where it can be readily accessed by the substrate. 


\section{CHAPTER 2}

\section{[}

EXPLORING THE LIGAND CHEMISTRY OF Pd MONOLAYER-PROTECTED [

CLUSTERS DURING SYNTHESIS IN THE PRESENCE OF OCTYLAMINE AND [

SUBSEQUENT EXPOSURE TO HYDROGEN, HEAT, AND THIOL PLACE[

\section{EXCHANGE REACTIONS}

\subsection{INTRODUCTION}

Here we describe the chemistry of octylamine ligands as stabilizers for Pd monolayerprotected clusters (MPCs). The octylamine ligands attach to the surface of the Pd as octanenitriles, as evidenced by infrared spectroscopy (IR), nuclear magnetic resonance spectroscopy (NMR), and gas chromatography-mass spectrometry (GC-MS). Upon exposure of the Pd MPCs in solution to $\mathrm{H}_{2}$ with dissolved $\mathrm{O}_{2}$, approximately $30 \%$ of the octanenitrile ligands are removed, the Pd MPCs aggregate, and some of the removed ligands convert into octanoic acid and octylamide. The ratio of octanitrile:octanoic acid:octanamide in solution is aproximately 20:10:70. Under a $\mathrm{N}_{2}$ atmosphere, exposure to $\mathrm{H}_{2}$ results in Pd MPC aggregation and only octanenitrile being removed from the surface, showing that dissolved oxygen is involved in the acid and amide formation. When the Pd MPCs are heated to $100{ }^{\circ} \mathrm{C}$ in solution, the Pd MPCs aggregate and $40 \%$ of the octanenitrile is removed from the surface and remains as octanenitrile in solution. Place-exchange of the octanenitrile ligands of the Pd MPCs with hexanethiol ligands results in the removal of the ligands as octanenitrile, octanoic acid, and octanamide in the solution in a 95:1:3 ratio. When place-exchange of the octanenitrile ligands occurs under an inert $\mathrm{N}_{2}$ atmosphere, the ligands come off the Pd as only octanenitrile. Here we propose reaction mechanisms for this interesting ligand chemistry. 


\subsection{EXPERIMENTAL}

Chemicals. Potassium tetrachloropalladate was purchased from Strem chemicals $(99.9 \%)$ and used as received. Toluene (99.9\%), Acetone (99.5\%) and Acetonitrile (99.9\%) were purchased from Sigma-Aldrich and used as received. Octylamine (99\%) and Sodium Borohydride (98\%) was purchased from Alfa Aesar Co. and used as received. Absolute Ethanol was purchased from Pharmco-Aaper and used as received. Chloroauric acid was synthesized by the dissolution of elemental gold (99.99\%) in aqua regia $(3: 1$ Hydrochloric Acid:Nitric Acid) followed by distillation and crystallization to result in sold $\mathrm{HAuCl}_{4} \cdot 3 \mathrm{H}_{2} \mathrm{O}$. Octanenitrile, octanoic acid, and octanamide were purchased from VWR Scientific. Deuterated chloroform (99.5\% atom purity) was obtained from Cambridge Isotope Laboratories. Barnstead nanopure water (18 $\mathrm{M} \Omega-\mathrm{cm})$ was used for all aqueous solutions.

Synthesis of Pd MPCs in the presence of octylamine. The Pd MPCs were prepared by a modified version of the Brust reaction ${ }^{53}$ as described by Ibanez et al. ${ }^{60}$ Briefly, $\sim 0.5 \mathrm{~g}$ $\mathrm{K}_{2} \mathrm{PdCl}_{4}$ was dissolved in $10 \mathrm{~mL}$ of $18 \mathrm{M} \Omega-\mathrm{cm}$ water and combined with $40 \mathrm{~mL}$ of toluene containing 1.5 equivalents of tetra n-octyl ammonium bromide (1.25g) with rapid stirring (the container in which the $\mathrm{K}_{2} \mathrm{PdCl}_{4}$ was dissolved was rinsed twice with $10 \mathrm{~mL}$ each of $18 \mathrm{M} \Omega-\mathrm{cm}$ water, and each rinse added to the reaction flask to ensure quantitative transfer of the palladium salt). After full transfer of $\mathrm{PdCl}_{4}{ }^{2-}$ from water to toluene, as indicated by the orange color transferring from the water to the toluene layer, 12 equivalents $(2.37 \mathrm{~g}, 3.03 \mathrm{~mL})$ of $\mathrm{N}$-octylamine were then added. At this point the toluene solution became colorless. After $1 \mathrm{~h}, 10$ equivalents $(0.58 \mathrm{~g})$ of freshly prepared sodium 
borohydride in $10 \mathrm{~mL}$ of $18 \mathrm{M} \Omega-\mathrm{cm}$ water were added with continued rapid stirring, followed by an additional $10 \mathrm{~mL}$ rinse with $18 \mathrm{M} \Omega$-cm water, at which point the solution became a dark brown-black color, indicative of the formation of metallic MPCs. After 3 $\mathrm{h}$, the water layer was separated and the toluene removed by evaporation using a Buchi rotary evaporator. $100 \mathrm{~mL}$ of acetonitrile was added to the remaining thick, viscous solution and the solution was allowed to stand overnight in order to dissolve the free amine ligands and precipitate the solid Pd MPCs. The insoluble solid MPCs were collected on a glass frit and washed with $50 \mathrm{~mL}$ acetonitrile followed by two $25 \mathrm{~mL}$ washes with ethanol. The solid MPCs were allowed to dry before collecting with a spatula.

The synthesized MPCs were characterized by proton NMR spectroscopy, thermogravimetric analysis (TGA), UV-VIS spectroscopy and GC-MS. The data were consistent with that reported previously by Moreno et al. ${ }^{20}$ and Ibanez et al. ${ }^{60}$

Synthesis of Au MPCs in the presence of octylamine. $\sim 0.5 \mathrm{~g} \mathrm{HAuCl}_{4} 3 \mathrm{H}_{2} \mathrm{O}$ were dissolved in $10 \mathrm{~mL}$ of $18 \mathrm{M} \Omega-\mathrm{cm}$ water and combined with $40 \mathrm{~mL}$ of toluene containing 1.5 equivalents of tetra n-octyl ammonium bromide $(1.20 \mathrm{~g})$ with rapid stirring until complete transfer of $\mathrm{AuCl}_{4}{ }^{-}$from water to toluene, as indicated by the solution color. The water layer became colorless, and the toluene layer became a yellow color. Two washes of $10 \mathrm{~mL}$ each with $18 \mathrm{M} \Omega$-cm water were added to the vessel in which the $\mathrm{HAuCl}_{4}$ was dissolved and then added to the reaction flash to ensure quantitative transfer of the $\mathrm{AuCl}_{4}{ }^{-}$ to the reaction. 12 equivalents of liquid $\mathrm{N}$-octylamine $(2.28 \mathrm{~g}, 2.9 \mathrm{~mL})$ were injected into the reaction flask and the mixture stirred for $1 \mathrm{~h}$. Next, a freshly prepared solution of 10 equivalents $(0.56 \mathrm{~g})$ of sodium borohydride in $10 \mathrm{~mL}$ of nanopure water was added with 
continued stirring. The vessel which contained the $\mathrm{NaBH}_{4}$ was washed with an additional $10 \mathrm{~mL} 18 \mathrm{M} \Omega-\mathrm{cm}$ water, and this added to the solution. At this point the organic phase became a very dark black color, indicative of the formation of metallic MPCs. After $3 \mathrm{~h}$, the aqueous layer was removed and the toluene was evaporated using a Buchi rotary evaporator. The remaining thick, viscous solution was mixed with $100 \mathrm{~mL}$ acetonitrile and allowed to stand overnight, leading to precipitation of the Au MPCs. The insoluble $\mathrm{Au}$ MPCs were collected on a glass frit and washed with $50 \mathrm{~mL}$ of acetonitrile followed by two $25 \mathrm{~mL}$ washes with ethanol. They were allowed to dry and the solid collected with a spatula.

Hydrogen Exposure. $40 \mathrm{mg}$ of Pd MPCs were dissolved in $8 \mathrm{~mL}$ of either chloroform-D or toluene. The solution was stirred while $100 \% \mathrm{H}_{2}$ gas was bubbled through it at a flow rate of $8 \mathrm{~mL} / \mathrm{min}$ (as measured by a calibrated flow meter) for $3 \mathrm{~h}$, which led to noticeable precipitation and aggregation of the MPCs in solution. The resulting supernatant was separated from the solid MPCs by pipetting off the liquid layer and the liquid analyzed by GC-MS and NMR spectroscopy. The precipitated solid Pd was isolated by first centrifuging the remaining mixture. As much liquid as possible was pipetted off, and the precipitated palladium allowed to dry under ambient conditions overnight. The solid Pd was analyzed by thermogravimetric analysis (TGA).

A Pd MPC solution was also exposed to $\mathrm{H}_{2}$ under an inert $\mathrm{N}_{2}$ atmosphere. For these experiments, similar concentrations as above were used, however the vial was sealed with a rubber septum and oil bubbler. Initially, the solution was sparged with $\mathrm{N}_{2}$ at a flow rate of $20 \mathrm{~mL} / \mathrm{min}$ with stirring for $15 \mathrm{~min}$. After purging, $\mathrm{N}_{2}$ was flowed through the headspace above the solution at $20 \mathrm{~mL} / \mathrm{min}$ while $\mathrm{H}_{2}$ was bubbled through the solution at 
a flow rate of $8 \mathrm{~mL} / \mathrm{min}$ with stirring. The solution and $\mathrm{Pd}$ were analyzed as previously described.

Heating Experiments. $40 \mathrm{mg}$ of Pd MPCs were dissolved in $8 \mathrm{~mL}$ toluene. This solution was stirred while being heated in an oil bath to $110^{\circ} \mathrm{C}$ for approximately $3 \mathrm{~h}$, until noticeable aggregation and precipitation of the MPCs occurred. The resulting supernatant was removed by pipetting and analyzed by Gas Chromatography-Mass Spectrometry (GC-MS) and Nuclear Magnetic Resonance (NMR). The resulting solid was isolated by first centrifuging the remaining mixture. As much liquid as possible was pipetted off, and the precipitated Pd allowed to dry under ambient conditions overnight. The solid Pd was analyzed by TGA.

Thiol Place-Exchange Reactions. $40 \mathrm{mg}$ of Pd MPCs were dissolved in $4 \mathrm{~mL}$ of dichloromethane. The ligand content of this solution was calculated using TGA data and an equimolar amount of hexanethiol was added to the solution and stirred for $12 \mathrm{~h}$. After, $1 \mathrm{~mL}$ of ethanol was added to the solution and the solution was allowed to stand for $3 \mathrm{~h}$ to ensure complete precipitation of the Pd MPCs. The solid Pd MPCs were removed by filtration and the supernatant was analyzed by GC-MS.

Characterization. GC-MS data was obtained on an Agilent 7890 Gas Chromatograph with an Agilent 7963 mass selective detector (quadropole mass spectrometer) with a $30 \mathrm{~m} \mathrm{HP-5} \mathrm{ms}$ column. The injector was used in splitless mode with a temperature of $150^{\circ} \mathrm{C}$. The oven had an initial temperature of $50{ }^{\circ} \mathrm{C}$ held for 2 min, followed by a temperature ramp of $35^{\circ} \mathrm{C} / \mathrm{min}$ to $160^{\circ} \mathrm{C}$, followed by a ramp to $200{ }^{\circ} \mathrm{C}$ at a rate of $15^{\circ} \mathrm{C} / \mathrm{min}$. The autosampler was used with an injection size of $0.5 \mu \mathrm{L}$. 
Peaks were initially assigned by library searching, and confirmed by using pure samples of the suspected compounds.

NMR spectra were obtained on a Varian $400 \mathrm{MHz}$ spectrometer with a robotic sample changer. H-NMR spectra were obtained in deuterated chloroform (Cambridge Isotope Laboratories $99.8 \%$ isotope purity) using 16 scans. TGA data were obtained on a TA Instruments thermogravimetric analyzer.

\subsection{RESULTS AND DISCUSSION}

Synthesis of Pd MPCs in the presence of octylamine. As described by our group and others, we synthesized Pd MPCs by borohydride reduction of $\mathrm{PdCl}_{4}{ }^{2-}$ in the presence of alkylamines, which was octylamine in this case. Previously, Zhonping et al. ${ }^{97}$ measured the NMR spectra of Pd MPCs coated with dodecylamine, where they described the amine-Pd interaction as an amino group. Ibanez et al. ${ }^{60}$ showed the presence of a nitrile group in the IR spectrum of as-prepared Pd MPCs synthesized in the presence of alkylamines, suggesting that the amine attaches to the Pd as a nitrile. In this work, we used gas chromatography with mass spectrometry detection (GC-MS) to help determine the chemistry of the octylamine when stabilizing the Pd MPCs. The GC-MS of the Pd MPCs synthesized in the presence of octylamine and dissolved in $\mathrm{CDCl}_{3}$ displayed one peak as shown in Figure 2.1A., which is assigned as octanenitrile based on the MS spectral library. This shows that the resulting Pd MPCs are actually stabilized by octanenitrile $(\mathrm{C} 7 \mathrm{CN})$ ligands rather than the original octylamine $(\mathrm{C} 8 \mathrm{NH} 2)$ ligands used in the synthesis. This is confirmed by the mass spectrum (Figure 2.1B) as well as by IR 

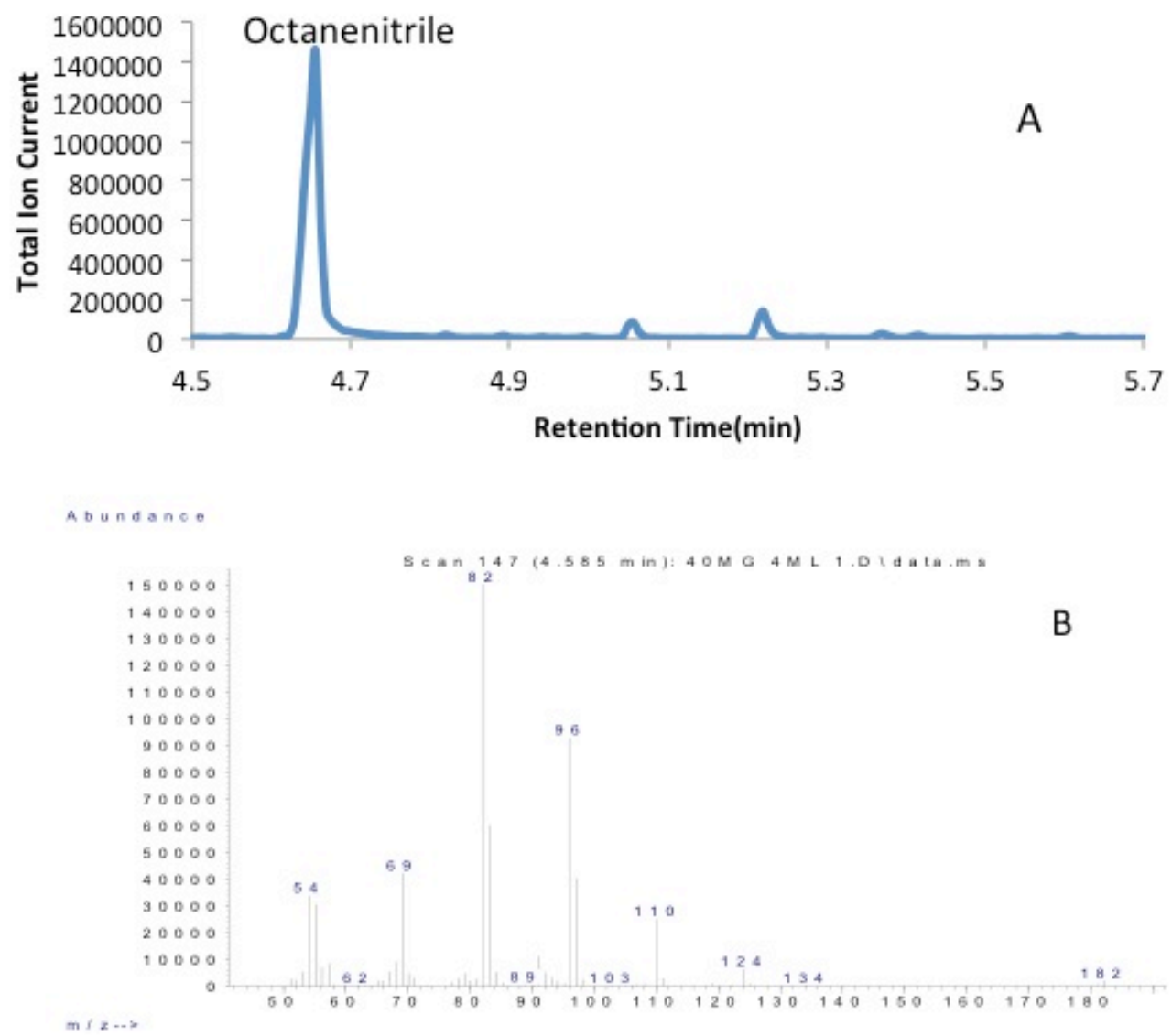

Figure 2.1. (A) GC-MS chromatogram of Pd MPCs synthesized in the presence of octylamine in $\mathrm{CDCl}_{3}$. (B) Mass Spectrum of observed octanenitrile peak.

The $\mathrm{C} 8 \mathrm{NH} 2$ ligands converted into $\mathrm{C} 7 \mathrm{CN}$ at some point during the reaction. 
spectroscopy. We believe that the amine ligands are initially adsorbed onto the Pd surface and the Pd catalyzes their oxidation to octanenitrile.

We monitored the reaction by obtaining GC-MS chromatograms at different times during the reaction as shown in Figure 2.2. After transferring $\mathrm{PdCl}_{4}{ }^{2-}$ to the toluene phase with tetraoctylammonium bromide, adding $\mathrm{C} 8 \mathrm{NH} 2$, and stirring for $45 \mathrm{~min}$, the GC-MS of the toluene phase shows one main peak in the chromatogram assigned as $\mathrm{C} 8 \mathrm{NH} 2$, showing no transformation at this stage (Figure 2.2, Frame A). Following the addition of aqueous $\mathrm{NaBH}_{4}$, a GC-MS of the toluene phase shows peaks for both $\mathrm{C} 8 \mathrm{NH} 2$ and $\mathrm{C} 7 \mathrm{CN}$, along with some boride related compounds (Figure 2.2, Frame B). This result shows that the $\mathrm{C} 7 \mathrm{CN}$ clearly formed during the sodium borohydride reduction step.

Characterization of the C7CN Pd MPCs showed a typical weight loss of $18 \pm 3 \%$ after ramping the temperature up to $800{ }^{\circ} \mathrm{C}$ by TGA. This weight loss corresponds directly to the amount of ligand initially present on the MPCs after synthesis and purification. An IR spectrum of the synthesized Pd MPCs showed the presence of a nitrile stretch at 2250 $\mathrm{cm}^{-1}$, consistent with that previously reported by Ibanez et al. ${ }^{60}$ The NMR spectrum of the Pd MPCs showed dramatically broadened peaks with no splitting, which confirms that the $\mathrm{C} 7 \mathrm{CN}$ ligands are bound to the Pd MPC core. In addition, the NMR spectrum of the Pd MPCs showed chemical shifts at $\delta 1.6$ and $\delta 2.4$, corresponding to the literature values for the $\beta$ and $\alpha$ carbons, respectively, of octanenitrile. Previous literature showed the size of Pd MPCs prepared the same way to be $\sim 3.0 \mathrm{~nm}$ in diameter based on transmission electron microscopy (TEM) images, although we did not measure the size in 
After adding $\mathrm{C} 8 \mathrm{NH} 2$
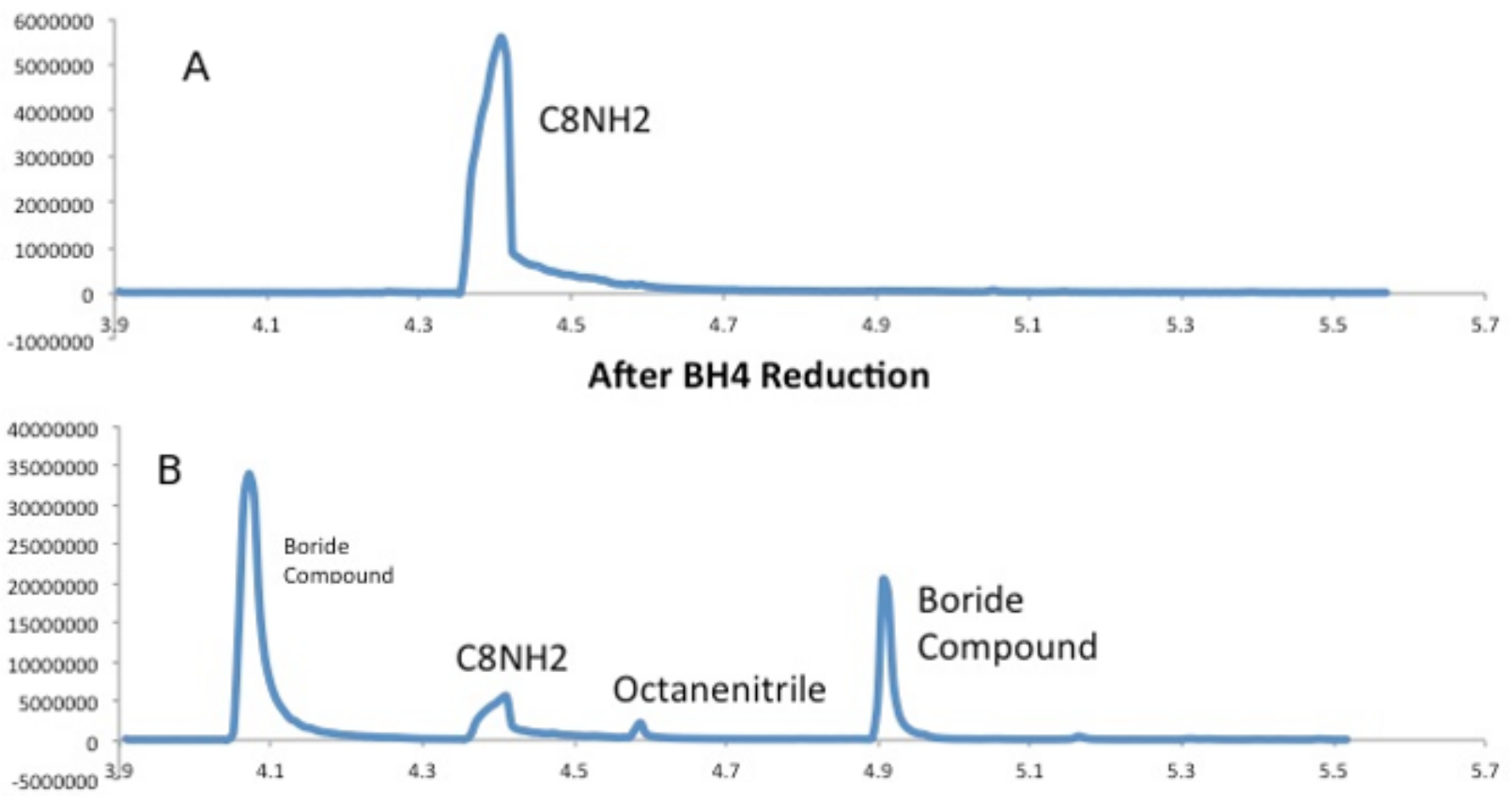

Figure 2.2. Reaction monitoring of the synthesis of C7CN Pd MPCs after (A) addition of $\mathrm{C} 8 \mathrm{NH} 2$ to $\mathrm{PdCl}_{4}{ }^{2-}$ and $\mathrm{TOABr}$ in toluene and (B) after subsequent reduction with $\mathrm{NaBH}_{4}$. 
this study. ${ }^{47}$ From the literature size value and our TGA, GC-MS, and spectroscopic data, we roughly estimate a composition of $\mathrm{Pd}_{1455} \mathrm{C}_{\mathrm{CN}} \mathrm{N}_{28}$ for these $\mathrm{Pd}$ MPCs.

Our previous work on Pd MPCs synthesized in the presence of octylamine described them as octylamine-coated $\mathrm{C} 8 \mathrm{NH} 2 \mathrm{Pd}$ MPCs. This is incorrect based on our new data, which consistently shows C7CN stabilizing the Pd MPCs. We will therefore refer to these MPCs as C7CN Pd MPCs throughout this thesis, accordingly. Interestingly, it was not possible to synthesize stable Pd MPCs through the direct addition of C7CN ligands during the synthesis. It is also a bit puzzling to form $\mathrm{C} 7 \mathrm{CN}$ from $\mathrm{C} 8 \mathrm{NH} 2$ ligands under reducing conditions, as $\mathrm{C} 7 \mathrm{CN}$ is more oxidized. Octanenitrile did not form in solution from $\mathrm{C} 8 \mathrm{NH} 2$ with the addition of sodium borohydride but no $\mathrm{PdCl}_{4}{ }^{2-}$, showing that $\mathrm{Pd}$ is also important (as a catalyst) for the reaction to occur. Although amines are known to act as reducing agents, ${ }^{98}$ we were not able to reduce $\mathrm{Pd}(\mathrm{II})$ to $\mathrm{Pd}(0)$ through the use of only C8NH2. Clearly there are still more details we need to learn regarding this interesting ligand transformation that occurs during Pd MPC synthesis with alkylamine stabilizers.

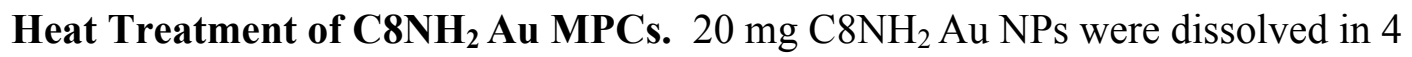
$\mathrm{mL}$ toluene. The solution was heated at $100{ }^{\circ} \mathrm{C}$ for $3 \mathrm{~h}$, at which point the solution became colorless and metallic gold was visibly present at the bottom of the vial. Analysis of the resultant supernatant by GC-MS showed that the removed ligands were primarily in the form of octylamine, as shown in Figure 2.3. This confirms that Pd metal specifically catalyzes the formation of the nitrile during synthesis.

Hydrogen treatment of C7CN Pd MPCs. When hydrogen is bubbled through a solution containing the C7CN Pd MPCs, the Pd MPCs begin to aggregate within a few seconds of the introduction of $\mathrm{H}_{2}$ as has been previously reported. ${ }^{2}$ This is presumably 


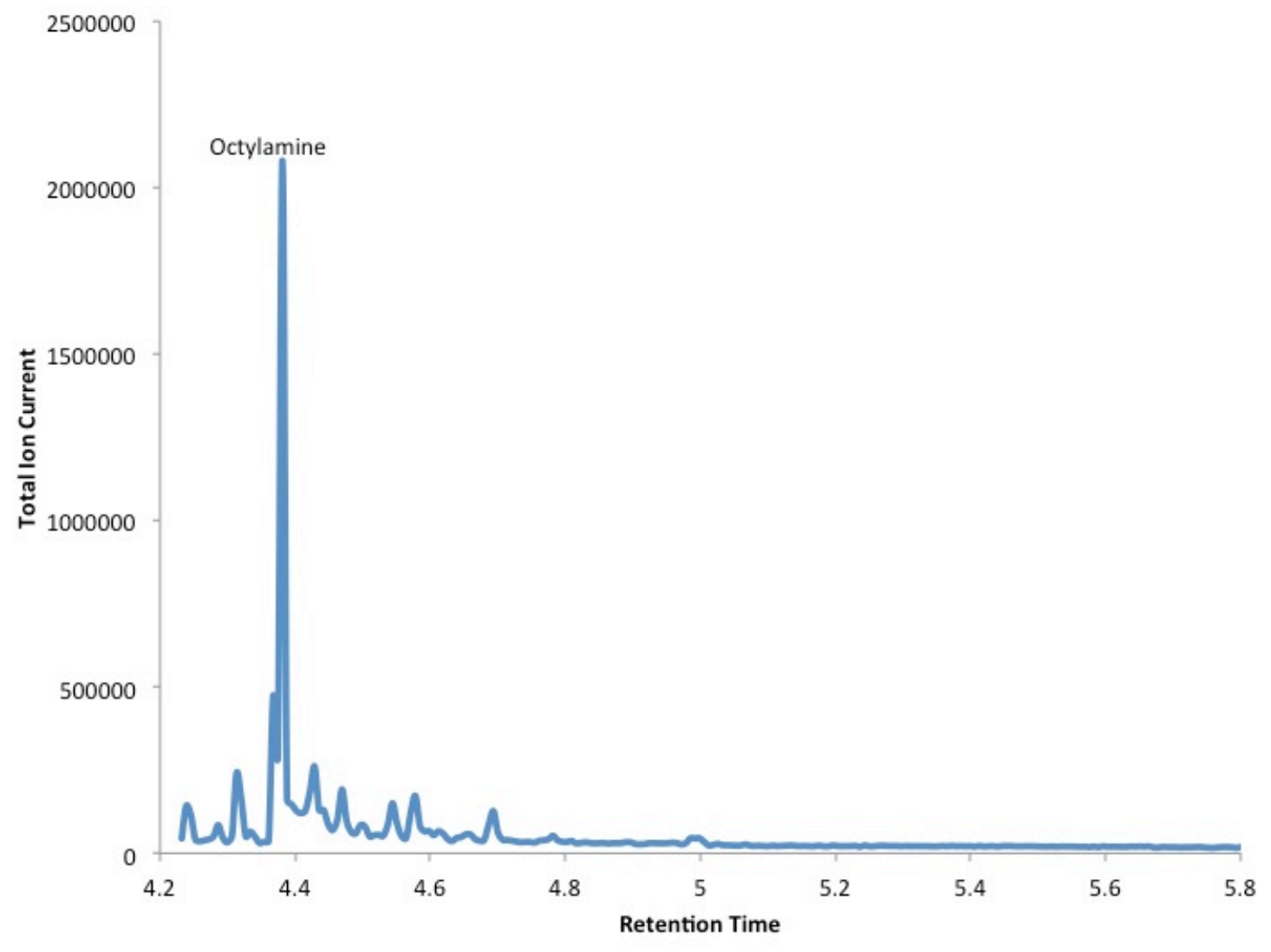

Figure 2.3: GC-MS Spectrum of $\mathrm{C} 8 \mathrm{NH} 2 \mathrm{Au}$ MPCs after heat treatment at $100{ }^{\circ} \mathrm{C}$ for $3 \mathrm{~h}$. 
due to the $\mathrm{PdH}_{\mathrm{x}}$ formation, which leads to aggregation through $\mathrm{PdH}_{\mathrm{x}}$ cross-linking or by replacement/removal of the $\mathrm{C} 7 \mathrm{CN}$ ligands during $\mathrm{PdH}_{\mathrm{x}}$ formation. ${ }^{99}$ Within 5 minutes of $\mathrm{H}_{2}$ bubbling, a change in color of the solution from brown to gray-black indicated Pd MPC aggregation. Within one hour, precipitated Pd appeared on the bottom of the reaction vessel and within 4 hours the solution was nearly colorless. The TGA data (Figure 2.4.) for the sample examined here shows a 50\% decrease in organic percentage after $\mathrm{H}_{2}$ exposure, showing that some ligands still remain on the surface of the Pd after aggregation and precipitation. The average composition(by TGA) is $18 \%+/-3 \%$ before treatment, and $13 \%+/-5 \%$ after treatment.

Specifically, as shown in Figure 2.4., C7CN Pd MPCs used in this particular sample are $25 \%$ organic before treatment with $\mathrm{H}_{2}$, and $13 \%$ after treatment with hydrogen, showing a loss of about one half the original ligands during $\mathrm{H}_{2}$ induced aggregation/precipitation. Analysis of the resulting solution remaining above the precipitated Pd metal by GC-MS shows the presence of octanenitrile, octanoic acid, and octanamide as show in Figure 2.5. Based on the TGA data, $40 \mathrm{mg}$ of C7CN Pd MPCs contain $5.3 \times 10^{-5}$ mol ligand, of which $3.3 \times 10^{-5}$ mol remained on the nanoparticles after aggregation and precipitation. $1.9 \times 10^{-5} \mathrm{~mol}$ were observed in the GC-MS spectrum, of which $4.5 \times 10^{-6} \mathrm{~mol}$ were octanenitrile, amounting to $24 \%, 5.6 \times 10^{-5} \mathrm{~mol}$ were octanoic acid, amounting to $30 \%$, and $8.6 \times 10^{-6}$ mol were octanamide, amounting to $46 \%$. The TGA and GC-MS data after $\mathrm{H}_{2}$ exposure accounted for $98 \%$ of the mols initially present on the Pd MPCs. These calculations were based on calibration curves of the three products using serial dilutions injected into the GC-MS. The concentration of the ligands, 

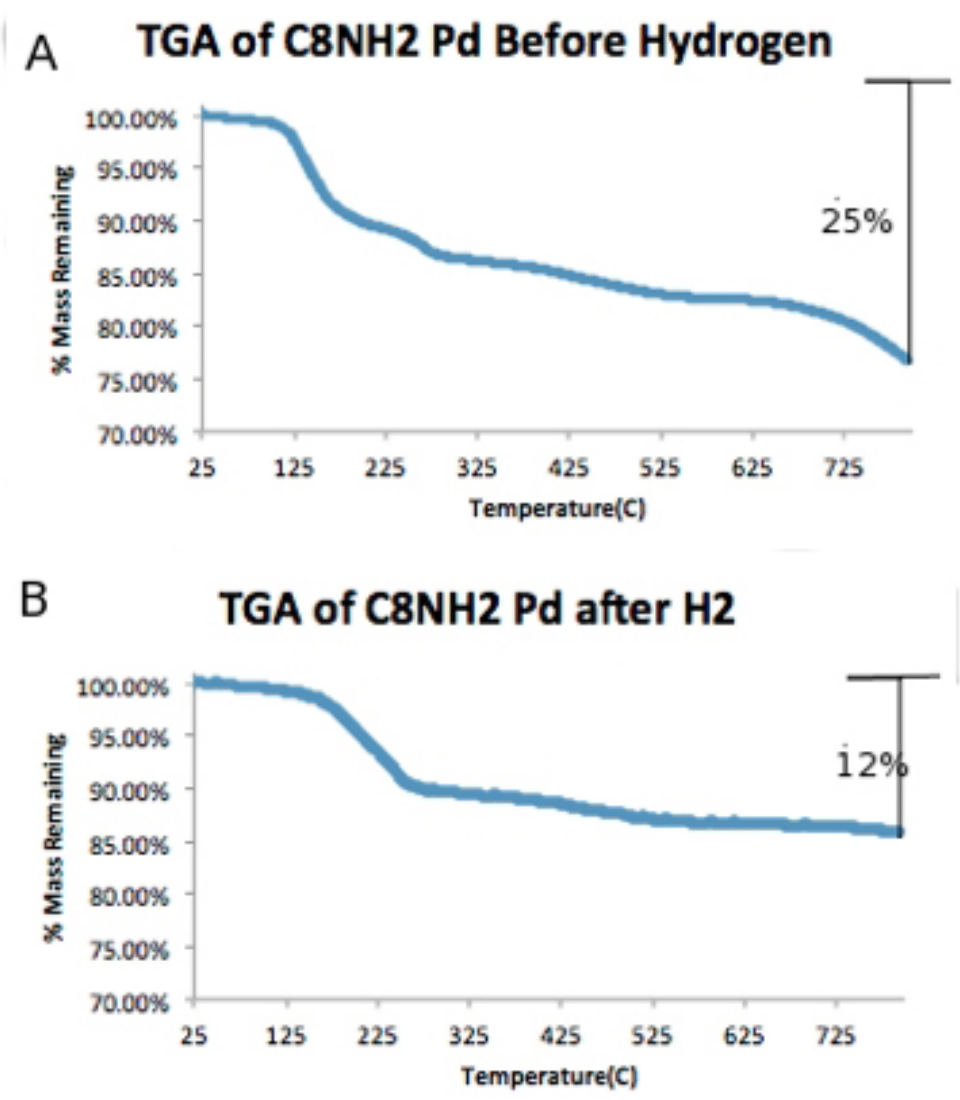

Figure 2.4. Thermogravimetric Analysis of C7CN Pd MPCs (A) Before $\mathrm{H}_{2}$ and (B) After $\mathrm{H}_{2}$ exposure for $4 \mathrm{~h}$. 


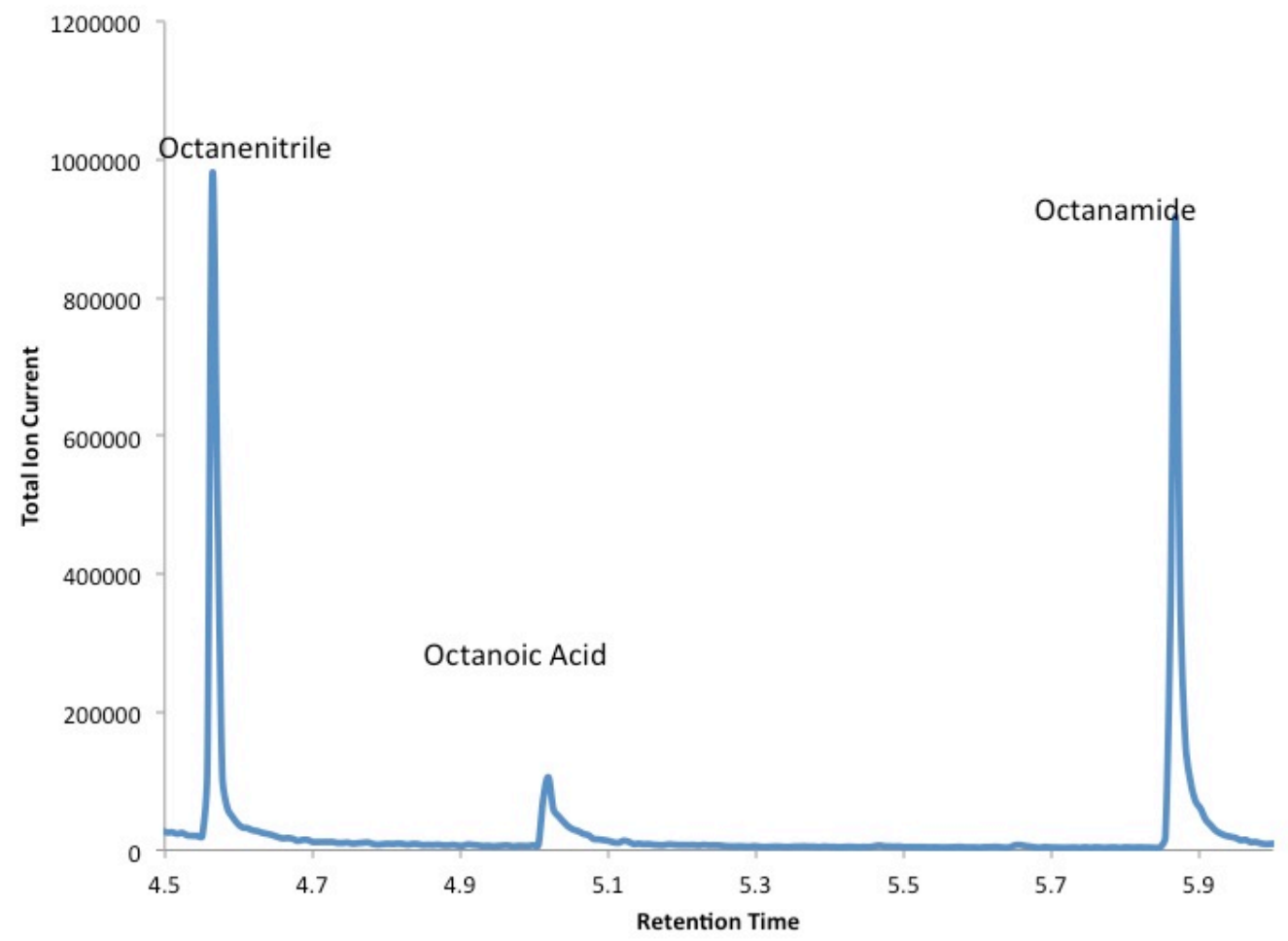

Figure 2.5. GC-MS Spectrum of supernatant of C7CN Pd MPCs in $\mathrm{CH}_{2} \mathrm{Cl}_{2}$ after treatment with $\mathrm{H}_{2}$. 
and in turn the moles of ligand present, was calculated based on the calibration curves and the known volume of solution used for the reaction.

An NMR spectrum is also consistent with the formation of these products as shown in Figure 2.6. Specifically, the NMR spectrum of the supernatant after hydrogen exposure to C7CN Pd MPCs shows peaks which correspond to the $\alpha$ and $\beta$ carbons of the carboxylic acid and amide groups of octanoic acid and octanamide, respectively, along with the nitrile, consistent with the GC-MS data. Figure 2.7 shows a schematic of the formation of the nitrile during the synthesis.

Removal of $\mathrm{O}_{2}$ from the MPC solution by purging with $\mathrm{N}_{2}$, followed by bubbling $\mathrm{H}_{2}$ under a $\mathrm{N}_{2}$ atmosphere resulted in the aggregation and precipitation of Pd MPCs as before, but the ligands that are removed are different. In this case, only octanenitrile appears in the solution as shown in Figure 2.8 The GC-MS data showed a recovery of $1.8 \times 10^{-5} \mathrm{~mol}$ octanenitrile from $40 \mathrm{mg}$ MPCs while $3.3 \times 10^{-5} \mathrm{~mol}$ ligand (as per TGA) remained on the aggregated NPs, as consistent with the recovery observed for MPCs treated with $\mathrm{H}_{2}$ in the presence of $\mathrm{O}_{2}$. This shows that $\mathrm{O}_{2}$ is involved in the acid and amide formation, as a result of it being dissolved in the solution or adsorbed onto the surface of the Pd.

Shimizu et al. ${ }^{100}$ reported that $\mathrm{Ag}$ oxide NPs supported on $\mathrm{SiO}_{2}$ can catalyze the formation of a variety of amides, including octanamide, from the respective nitrile. Using this reaction as a model, we surmise that there exists $\mathrm{PdO}$ on the surface of the as prepared C7CN Pd NPs. This is shown schematically in Figure 2.9. With the introduction of $\mathrm{H}_{2}$, the combined formation of surface $\mathrm{PdH}$ and $\mathrm{PdO}$ results in an $\mathrm{OH}$ group which can attach to the nitrile, resulting in the octanamide. The acid is formed through additional 


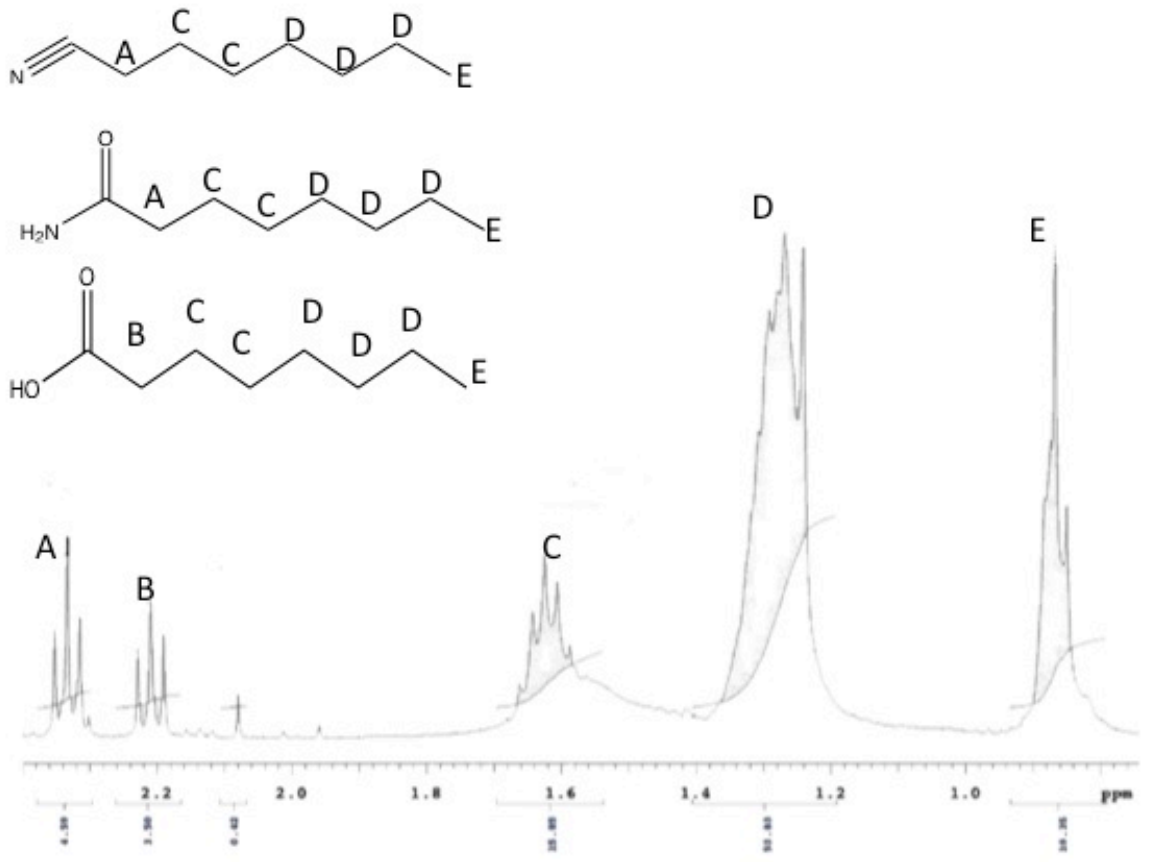

Figure 2.6. NMR spectrum of the supernatant of a solution of $\mathrm{C} 7 \mathrm{CN} \mathrm{Pd}$ nanoparticles treated with $8 \mathrm{~mL} / \mathrm{min}$ of $\mathrm{H}_{2}$ gas for $3 \mathrm{~h}$. 


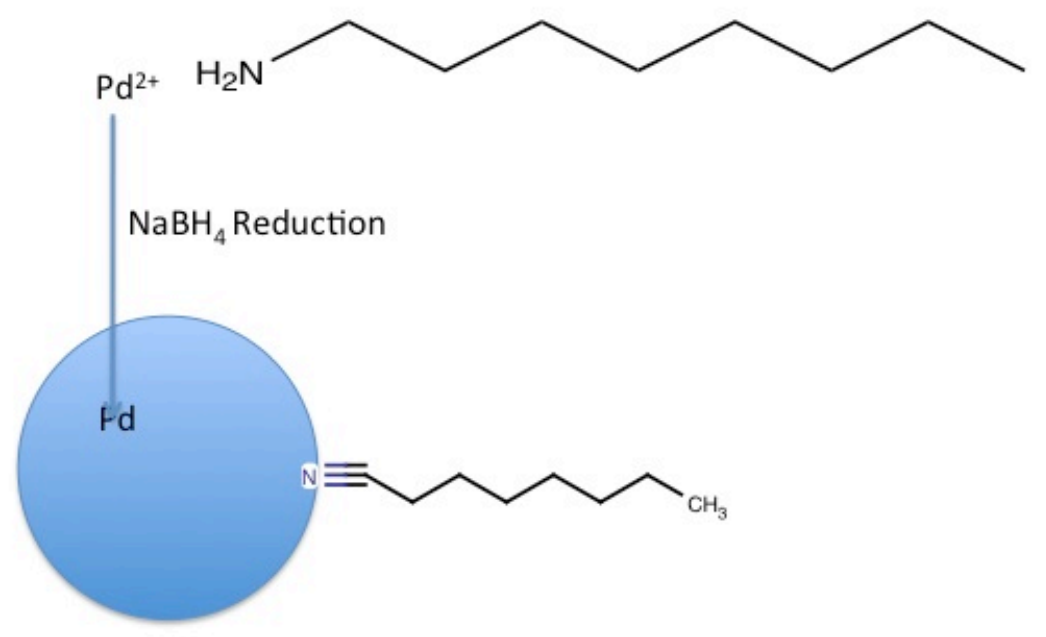

Figure 2.7. Schematic of the formation of the nitrile on the Pd surface during the synthesis of Pd MPCs in the presence of octylamine. 


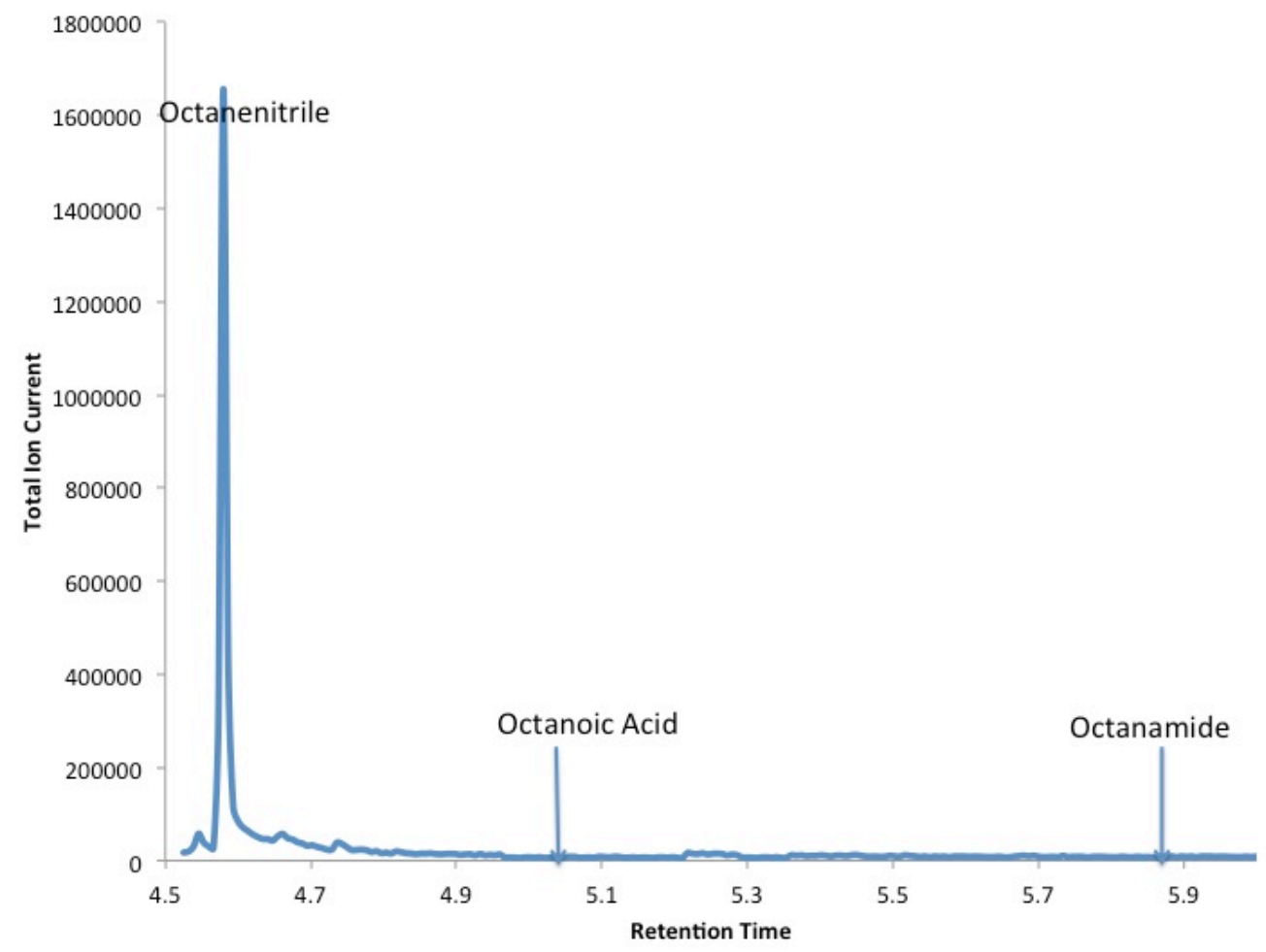

Figure 2.8. GC-MS spectrum of the supernatant of C7CN MPCs treated with $\mathrm{H}_{2}$ in the absence of $\mathrm{O}_{2}$. 


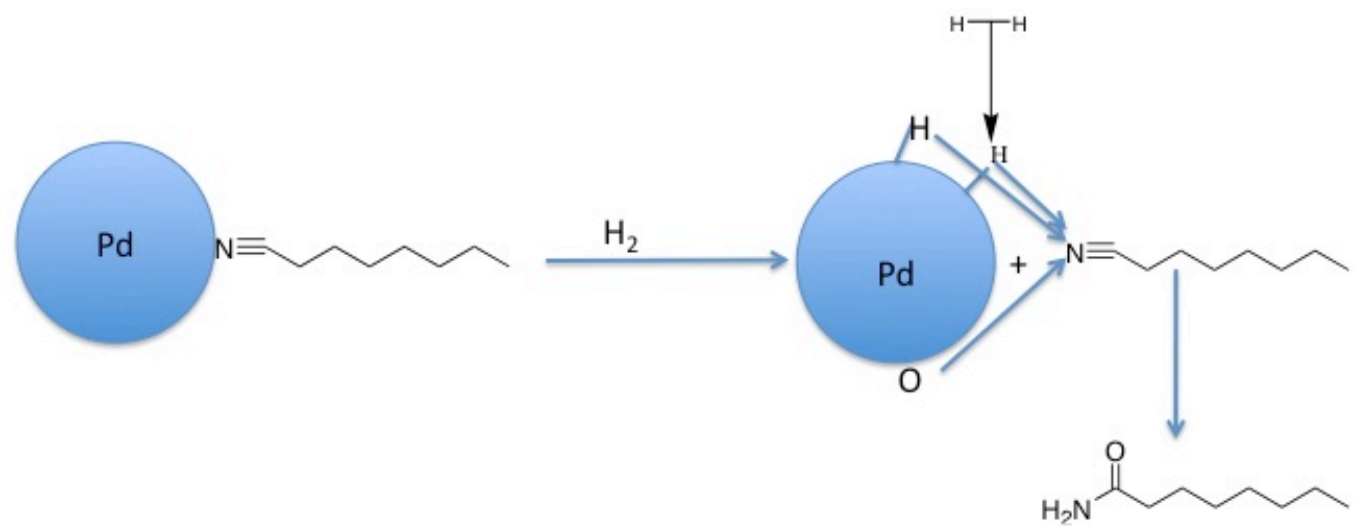

Figure 2.9. Mechanism for the formation of amide from nitrile. 
oxidation of the amide by $\mathrm{O}$ and $\mathrm{H}$ adsorbed onto the Pd surface. This is shown schematically in Figure 2.10

Heat treatment of C7CN Pd MPCs. We also treated samples of C7CN Pd MPCs at high temperature, as many catalysis applications involve reactions at elevated temperatures. We therefore wanted to better understand the C7CN Pd MPC stability and ligand transformations that potentially occur at high temperature. When a solution of $\mathrm{C} 7 \mathrm{CN}$ Pd MPCs was heated to $100^{\circ} \mathrm{C}$ or higher, aggregation and precipitation of the $\mathrm{Pd}$ MPCs occurred within $1 \mathrm{~h}$. Analysis of the supernatant by GC-MS showed the presence of only octanenitrile, as shown in Figure 2.11. For a $40 \mathrm{mg}$ sample of MPCs, we measured $2.3 \times 10^{-5} \mathrm{~mol}$ octanenitrile ligand in the solution from GC-MS and $2.8 \times 10^{-5} \mathrm{~mol}$ remaining on the aggregated NPs by TGA, for a total of $5.1 \times 10^{-5} \mathrm{~mol}$, which is very close to the total of $5.3 \times 10^{-5} \mathrm{~mol}$ of ligand that is initially coating $40 \mathrm{mg}$ of C7CN Pd MPCs. Importantly, the GC-MS and TGA accounted for almost all of the ligands. No effort was made to remove $\mathrm{O}_{2}$ from solution in these experiments as no octanoic acid or octanamide was observed. These results lead us to conclude that both $\mathrm{H}_{2}$ (and the resultant formation of $\mathrm{PdHx}$ ) and $\mathrm{O}_{2}$ are required for the formation of the acid and amide products. A proton source is clearly needed. In addition, Au MPCs coated by octylamine were not shown to form a nitrile group, but rather remained attached as amines. This shows that Pd is clearly necessary for the formation of the nitrile.

Ligand Place Exchange of C7CN with hexanethiol. Ligand place-exchange reactions on metal MPCs has been shown to be a very useful strategy to alter the chemical functionalization for a variety of fundamental and applied studies. This has 


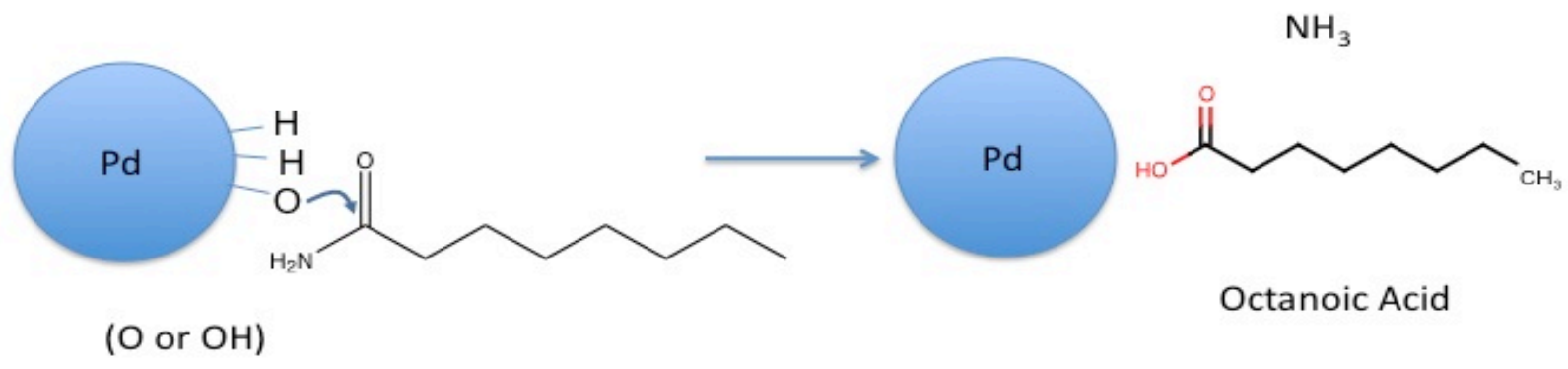

Figure 2.10. Formation of octanoic acid from octanamide. 


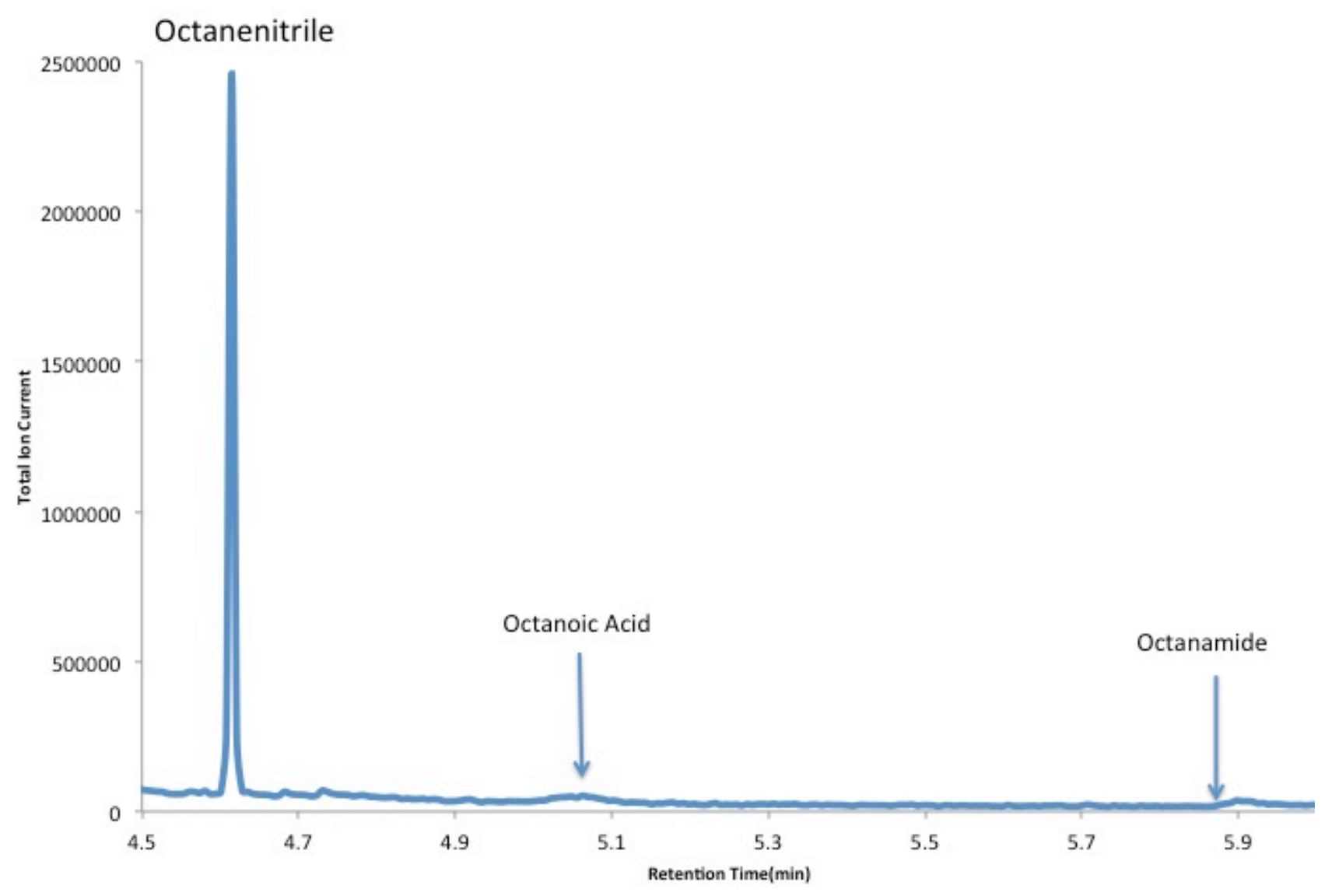

Figure 2.11. GC-MS spectrum of supernatant after heating Pd MPCs to $100{ }^{\circ} \mathrm{C}$ for $3 \mathrm{~h}$. 
been widely studied on Au MPCs especially, ${ }^{84}$ but also demonstrated with Pd MPCs. ${ }^{47}$ We therefore monitored the chemistry of C7CN ligands during removal from Pd by place-exchange with more strongly binding hexanethiol ligands. The strong affinity of thiol groups for Pd led to the removal of the $\mathrm{C} 7 \mathrm{CN}$ ligands from $\mathrm{Pd}$ and the binding of hexanethiol on the Pd as hexanethiolates. The GC-MS chromatogram of the solution after place-exchange with hexanethiol and precipitation of the Pd MPCs shows that the C7CN ligands came off the surface as octanoic acid, octanamide, and octanenitrile, just as it had during $\mathrm{H}_{2}$-induced ligand removal in the presence of $\mathrm{O}_{2}$ (Figure 2.12A). The $\mathrm{H}$ from the hexanethiol is assumed to be the H source. Although not explicitly illustrated, it can be surmised that the oxidation to octanoic acid proceeds in the same manner as in $\mathrm{H}_{2}$ treatment. Removal of $\mathrm{O}_{2}$ from this solution again results in only the formation of octanenitrile, as shown in Figure 2.12B. The formation of octanoic acid and octanamide occurs as a result of the $\mathrm{O}$ from $\mathrm{PdO}$ or adsorbed $\mathrm{O}_{2}$ and $\mathrm{H}$ from the incoming hexanethiol as the proton source. This is summarized in Figure 2.13. For a $40 \mathrm{mg}$ sample, approximately $69 \%$ of the hexanethiol ligands exchanged onto the Pd MPCs based on the fact that $3.7 \times 10^{-5} \mathrm{~mol}$ of $\mathrm{C} 7 \mathrm{CN}$ and $\mathrm{C} 7 \mathrm{CN}$-derived ligands were measured in solution out of the original $5.3 \times 10^{-5} \mathrm{~mol}$. From the $3.7 \times 10^{-5}$ mol total, there was $2.2 \times 10^{-5} \mathrm{~mol}$ octanenitrile $(61 \%), 6.3 \times 10^{-6} \mathrm{~mol}$ octanoic acid $(17 \%)$, and $8.2 \times 10^{-6}$ mol octanamide $(22 \%)$

The presence of $\mathrm{O}_{2}$ and the thiol $\mathrm{SH}$ as a proton source leads to the formation of octanoic acid and octanamide, catalyzed by $\mathrm{Pd}$ or $\mathrm{PdO}$. In the absence of $\mathrm{O}_{2}$, only octanenitrile is present. 

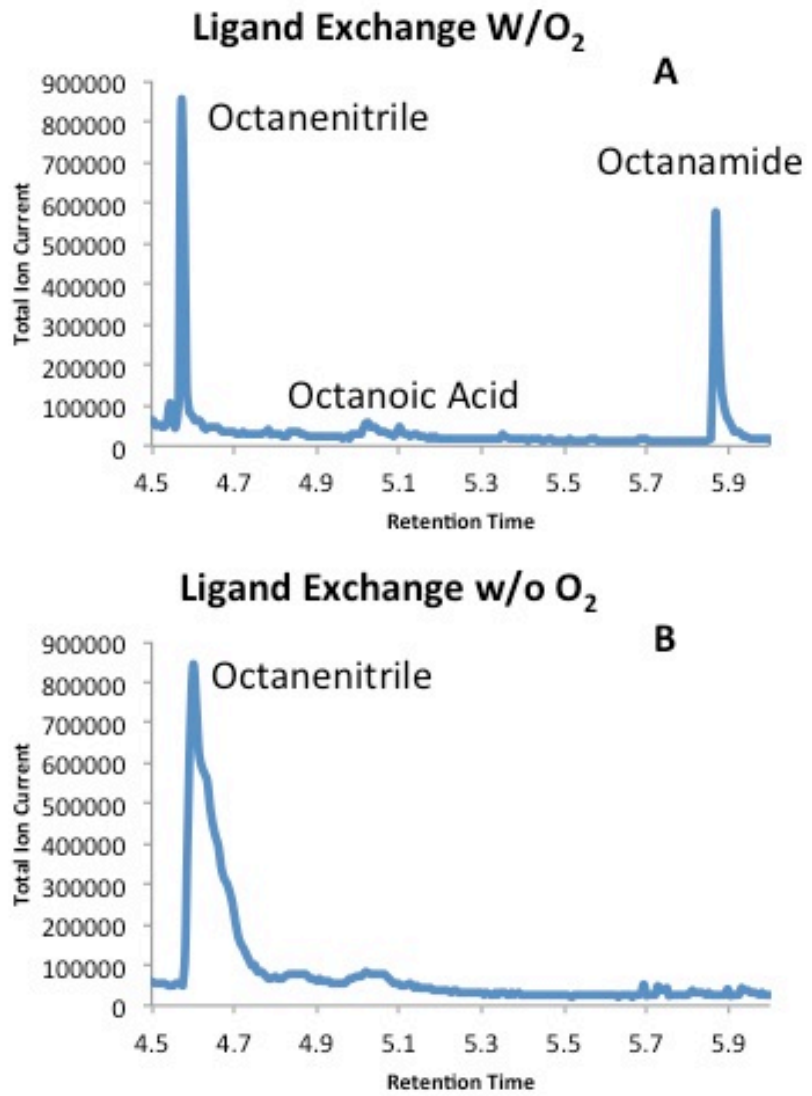

Figure 2.12. GC-MS chromatograms of supernatant of C7CN Pd MPCs after placeexchange with hexanethiol $(\mathrm{C} 6 \mathrm{SH})$ in the presence $(A)$ and absence (B) of $\mathrm{O}_{2}$. Pd MPCs were precipitated following place-exchange 

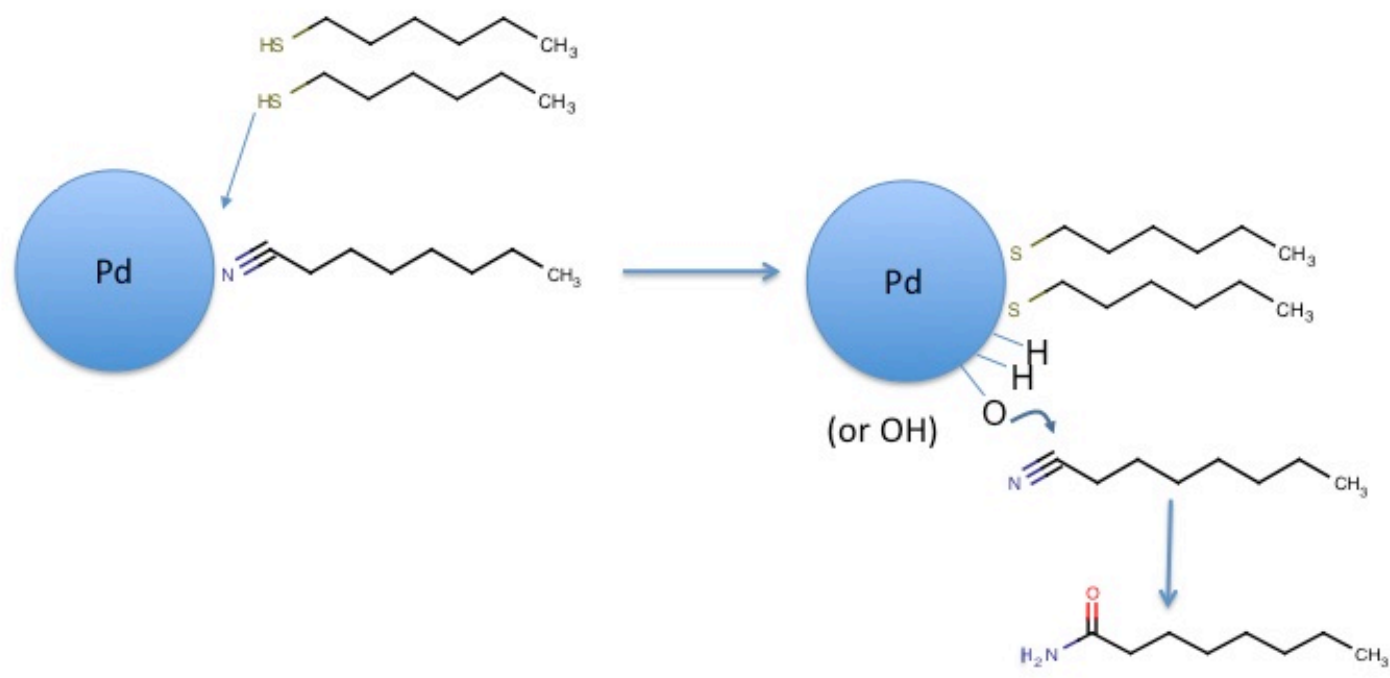

Octanamide

Figure 2.13. Proposed mechanism of octanoic acid and octanamide formation during ligand exchange. 


\subsection{CONCLUSIONS}

We have synthesized Pd MPCs by reduction of $\mathrm{PdCl}_{4}{ }^{2-}$ with sodium borohydride in the presence of $\mathrm{C} 8 \mathrm{NH} 2$, showing that $\mathrm{C} 8 \mathrm{NH} 2$ is oxidized to $\mathrm{C} 7 \mathrm{CN}$ during the reduction of $\mathrm{PdCl}_{4}{ }^{2-}$ to $\mathrm{Pd}^{0}$ metal, leading to the synthesis of $\mathrm{C} 7 \mathrm{CN}$-coated Pd MPCs. When the C7CN Pd MPCs are exposed to $\mathrm{H}_{2}$ gas in the presence of ambient $\mathrm{O}_{2}$, the Pd MPCs aggregate and precipitate as shown previously. What we discovered new in this report is that the C7CN Pd MPCs lose about half of their ligands and they come off the Pd as a mixture of octanenitrile $(\mathrm{C} 7 \mathrm{CN})(29 \%)$, octanoic acid $(\mathrm{C} 7 \mathrm{COOH})(15 \%)$ and octanamide $(\mathrm{C} 7 \mathrm{CONH} 2)(70 \%)$. The octanamide and octanoic acid are not formed when $\mathrm{O}_{2}$ is removed from the solution before and during $\mathrm{H}_{2}$ exposure, showing that $\mathrm{O}_{2}$ plays a large role in the transformation. Only $\mathrm{C} 7 \mathrm{CN}$ comes off of the Pd MPCs when the ligands are removed from the Pd MPCs by heating, showing that the acid and amide products require a H source. When Au MPCs synthesized in the presence of octylamine are heated, only octylamine comes off of the MPCs. This shows that Pd specifically catalyzes nitrile formation during the synthesis, which is not possible with Au. During ligand placeexchange reactions between C7CN Pd MPCs and hexanethiol in the presence of $\mathrm{O}_{2}$, the C7CN ligands are again removed as a mixture of octanenitrile (60\%), octanoic acid $(17 \%)$ and octanamide $(22 \%)$, where the proton on the thiol $(\mathrm{SH})$ serves as the proton source. The $\%$ of octanamide in this case is $22 \%$ as compared to $70 \%$ when $\mathrm{H}_{2}$ is used, showing that $\mathrm{H}_{2}$ is a better $\mathrm{H}$ source for amide formation. In the absence of $\mathrm{O}_{2}$, the ligands remain as octanenitrile during place exchange, similar to $\mathrm{H}_{2}$ exposure in the absence of $\mathrm{O}_{2}$. 
All of the reactions are summarized in Table 1. The results reveal important information about the ligand chemistry of Pd MPCs synthesized in the presence of alkylamines, which have been shown to be useful in catalysis and sensing applications, and likely others in the future. ${ }^{70,101}$ It is important to gain a better fundamental understanding about the ligand chemistry during these common Pd MPC reactions relevant to catalysis. Further studies will examine if it is possible to more fully utilize the reactions presented herein for larger scale catalytic reactions to purposely convert amines or nitriles into amides or acids, as well as further examine the mechanisms of the reactions. 
Table 1: Reaction products and recovery for different treatments.

\begin{tabular}{|c|c|c|c|c|c|c|c|c|}
\hline & & \begin{tabular}{|l|} 
Initial \\
Mols \\
Present in \\
$40 \mathrm{mg}$ \\
Sample \\
Before \\
\end{tabular} & $\begin{array}{l}\text { TGA \% } \\
\text { Organic } \\
\text { Before } \\
\end{array}$ & $\begin{array}{l}\text { TGA \% } \\
\text { Organic } \\
\text { After }\end{array}$ & \begin{tabular}{|l} 
After \\
Moles \\
Ligand
\end{tabular} & $\begin{array}{c}\text { Octane } \\
\text {-nitrile }\end{array} \mid$ & $\left|\begin{array}{l}\text { Octanic } \\
\text { Acid }\end{array}\right|$ & $=\begin{array}{l}\text { Octan } \\
\text { amide }\end{array}$ \\
\hline & $\begin{array}{l}\text { With } \\
\mathrm{O}_{2}\end{array}$ & $\begin{array}{l}5.3 \times 10^{-5} \\
\mathrm{~mol}\end{array}$ & $\begin{array}{l}18 \%+/- \\
3 \%\end{array}$ & \begin{tabular}{|l}
$13 \%+/-$ \\
$5 \%$ \\
$3.84 \times 10^{-5}$ \\
mol \\
\end{tabular} & $1.8 \times 10^{-5}$ & $\begin{array}{l}29 \%+/ \\
-7 \%\end{array}$ & $\begin{array}{l}15 \%+/ \\
-10 \%\end{array}$ & $\begin{array}{l}55 \%+ \\
/-8 \%\end{array}$ \\
\hline $\mathrm{H}_{2}$ & $\mathrm{No} \mathrm{O}_{2}$ & $\begin{array}{l}5.3 \times 10^{-5} \\
\mathrm{~mol}\end{array}$ & $\begin{array}{l}18 \%+/- \\
3 \%\end{array}$ & \begin{tabular}{|l}
$13 \%+/-$ \\
$5 \%$ \\
$3.84 \times 10^{-5}$ \\
mol \\
\end{tabular} & $1.8 \times 10^{-5}$ & $100 \%$ & $0 \%$ & $0 \%$ \\
\hline Heat & $\begin{array}{l}\text { With } \\
\mathrm{O}_{2}\end{array}$ & $\begin{array}{l}5.3 \times 10^{-5} \\
\mathrm{~mol}\end{array}$ & $\begin{array}{l}18 \%+/- \\
3 \%\end{array}$ & \begin{tabular}{|l|}
$11 \%+/-$ \\
$5 \%$ \\
$3.23 \times 10^{-5}$ \\
mol \\
\end{tabular} & $2.3 \times 10^{-5}$ & $100 \%$ & $0 \%$ & $0 \%$ \\
\hline & $\mathrm{No}_{2}$ & & --- & -- & - & --- & --- & -- \\
\hline Ligand & \begin{tabular}{|l|} 
With \\
$\mathrm{O}_{2}$
\end{tabular} & $\begin{array}{l}.3 \times 10^{-5} \\
\mathrm{~mol}\end{array}$ & $\begin{array}{l}18 \%+/- \\
3 \%\end{array}$ & --- & $3.6 \times 10^{-5}$ & $60 \%$ & $17 \%$ & $22 \%$ \\
\hline $\begin{array}{l}\text { Excn- } \\
\text { ange }\end{array}$ & $\mathrm{NoO}_{2}$ & $\begin{array}{l}5.3 \times 10^{-5} \\
\text { mol }\end{array}$ & $\begin{array}{l}18 \%+/- \\
3 \%\end{array}$ & -- & $2.6 \times 10^{-5}$ & $100 \%$ & $0 \%$ & $0 \%$ \\
\hline
\end{tabular}


CHAPTER 3

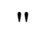

INVESTIGATION OF RELATIVE CATALYTIC EFFICIENCY OF PALLADIUM(]

SILVER ALLOY MPCs PREPARED BY CO-REDUCTION AND BY GALVANIC

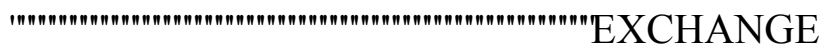

\subsection{INTRODUCTION}

Here, we examine the relative catalytic properties of C12S-stabilized MPCs with PdAg alloy cores. Alloy MPCs were prepared by two different techniques. In coreduction, salts of both metals are mixed together and simultaneously reduced. In galvanic exchange, MPCs of one metal (in this case Ag) are first prepared, and then, through a galvanic exchange process, atoms of a second metal (Pd) are deposited on the surface by replacing the first metal. It was hoped that by having more of the catalytic metal $(\mathrm{Pd})$ on the surface, the resulting MPCs would be more catalytically active in the hydrogenation and isomerization of allyl alcohol. The results were inconclusive, however.

\subsection{EXPERIMENTAL}

Preparation of PdAg MPCs by co-reduction. $\sim 0.5 \mathrm{~g} \mathrm{~K}_{2} \mathrm{PdCl}_{4}$ was first dissolved in $10 \mathrm{~mL} 18 \mathrm{M} \Omega$-cm water. Next, 1.5 equivalents $(1.25 \mathrm{~g})$ of the TOABr phase transfer reagent was separately dissolved in $40 \mathrm{~mL}$ of toluene. The aqueous $\mathrm{K}_{2} \mathrm{PdCl}_{4}$ solution was then added to the toluene solution of TOABr followed by two rinses of the container with $5 \mathrm{~mL}$ each of $18 \mathrm{M} \Omega$-cm water. The water layer was removed. $\mathrm{AgCF}_{3} \mathrm{O}_{3} \mathrm{~S}$ (Silver Triflate) was then added to the toluene layer in an amount that will give the desired Pd:Ag ratio. After complete dissolution of the silver triflate in toluene, the appropriate 
amount of ligand was added in 0.5 equivalents of dodecanethiol ligands, and 12 equivalents for hexadecylamine ligands, taking into account the total mols of metal present in the reaction. The metal ions were then reduced using 10 equivalents of $\mathrm{NaBH}_{4}$. After $3 \mathrm{~h}$, the water layer was removed. The toluene was then removed using a Rotary Evaporator $\left(40^{\circ} \mathrm{C}\right.$ water bath, 16 millibar pressure $)$. After complete removal of the toluene, the MPCs were suspended in $100 \mathrm{~mL}$ acetonitrile (in which they were not soluble) and allowed to stand overnight. The MPCs were collected over a glass frit and washed twice with $20 \mathrm{~mL}$ acetone and $20 \mathrm{~mL}$ ethanol. They were allowed to dry on the frit and stored under ambient conditions in a sealed glass vial. The resulting MPCs were characterized by UV-Vis and TGA. They were also evaluated for $\mathrm{H}_{2}$ stability and catalytic performance in the hydrogenation/isomerization of ally alcohol.

Preparation of Ag MPCs. $\sim 0.5 \mathrm{~g} \mathrm{AgNO}_{3}$ were first dissolved in $10 \mathrm{~mL} 18 \mathrm{M} \Omega-\mathrm{cm}$ water. 1.5 eq $\mathrm{TOABr}(2.41 \mathrm{~g})$ were dissolved in $40 \mathrm{~mL}$ toluene. The $\mathrm{AgNO}_{3}$ solution was was added to the toluene/TOABr solution and stirred for $20 \mathrm{~min}$. The water layer was then removed, and $0.5 \mathrm{eq}(0.298 \mathrm{~g}, 352 \mu \mathrm{L})$ of dodecanethiol ligand added. This was allowed to stir for 45 min under ice, and the solution was reduced at room temperature for $3 \mathrm{~h}$ using 10 equivalents of $\mathrm{NaBH}_{4}(1.11 \mathrm{~g})$ in $10 \mathrm{~mL}$ of $18 \mathrm{M} \Omega$-cm water. The water layer was removed in a separatory funnel, and the toluene removed using a rotary evaporator $\left(40^{\circ} \mathrm{C}\right.$ bath temperature, 16 millibar pressure). The MPCs were suspended in $100 \mathrm{~mL}$ acetonitrile overnight, then collected over a glass frit. The MPCs were washed twice with $20 \mathrm{~mL}$ acetone, followed by washing twice with $20 \mathrm{~mL}$ ethanol. The resulting nanoparticles were dried, evaluated for $\mathrm{H}_{2}$ stability, and characterized using UV-VIS and TGA. 
Galvanic Exchange of Ag MPCs. The Ag MPCs were weighed and dissolved in $~ 10$ $\mathrm{mL}$ toluene. An appropriate amount of $\mathrm{K}_{2} \mathrm{PdCl}_{4}$ - to give the desired $\mathrm{PdAg}$ ratio while also bearing in mind that each Pd replaces two Ag atoms - was dissolved in $10 \mathrm{~mL} 18$ $\mathrm{M} \Omega-\mathrm{cm}$ water. The solutions were mixed, then stirred overnight under ambient conditions. The water layer was removed by pipetting, and the toluene removed using a rotary evaporator $\left(40^{\circ} \mathrm{C}\right.$ bath temperature, 16 millibar pressure). The MPCs were suspended in $50 \mathrm{~mL}$ acetonitrile and allowed to stand overnight. The MPCs were collected over a glass frit and washed twice with $20 \mathrm{~mL}$ acetone, followed by washing twice with $20 \mathrm{~mL}$ ethanol. The resulting MPCs were dried, and then evaluated for $\mathrm{H}_{2}$ stability and catalytic activity. The MPCs were characterized using UV-VIS and TGA.

Characterization of MPCs by UV-VIS. When evaluating mixed-metal MPCs, a UV-VIS spectrum provides important information. $\mathrm{Pd}^{0}$ displays no plasmon band in the visible range, while $\mathrm{Ag}^{0}$ displays a plasmon band at $\sim 415 \mathrm{~nm}$, allowing qualitative evaluation of the Ag and Pd present in the MPC. More importantly, for galvanic exchange reactions, the loss of the Ag plasmon band in the UV-VIS spectrum would be indicative of successful galvanic exchange.

Evaluating Hydrogen Stability of MPCs. Because $\mathrm{H}_{2}$ is involved in all the catalytic reactions studied herein, evaluating the $\mathrm{H}_{2}$ stability of MPCs was an important first step. A solution of MPCs in toluene was prepared in a quartz cuvette, and the concentration adjusted until a peak absorbance between 0.8 and 1.0 was obtained. The solution level in the cuvette was noted. $\mathrm{H}_{2}$ gas was then bubbled through the solution in the cuvette at a rate of $8 \mathrm{~mL} / \mathrm{min}$. The UV-VIS absorbance was then monitored after bubbling $\mathrm{H}_{2}$ for a total of $5 \mathrm{~min}, 15 \mathrm{~min}, 30 \mathrm{~min}$, and $60 \mathrm{~min}$. Before each reading, the level of toluene was 
topped off to the original level, and then briefly bubbled with $\mathrm{H}_{2}$ to ensure complete mixing. Figure 3.1 shows the experimental set-up for this analysis (Frame A), a typical UV-Vis spectrum for an Ag MPC (Frame B), and the stability of these particles over time when treated with $\mathrm{H}_{2}$

For MPCs containing predominately Pd, the absorbance over time was monitored and plotted at $311 \mathrm{~nm}$. For those containing predominantly Ag, the peak absorbance of the plasmon band was used as reference point. As reported by Moreno, ${ }^{47}$ pure Pd and PdAg alloy MPCs prepared by co-reduction were $\mathrm{H}_{2}$ stable with thiol chain lengths as short as 6 carbons, while amine coated MPCs need a chain length of at least 16 carbons for $\mathrm{H}_{2}$ stability. As discussed in Chapter 2, Pd MPCs with 8-carbon amine chains are not $\mathrm{H}_{2}$ stable and were not used in this study.

It was found to be impossible to prepare even shelf-stable pure Ag MPCs with thiol chain lengths of less than 12 carbons. Because the Ag MPCs must be redissolved in toluene for the galvanic exchange reactions, it was found necessary to use $\mathrm{C} 12 \mathrm{~S} \mathrm{Ag}$ MPCs for galvanic exchange reactions. While this is a fairly long chain length, Moreno showed that the catalytic turnover frequency is not much lower than Pd MPCs coated with hexanethiol ligands. ${ }^{47}$ A spectrum and stability plot for C12S Pd:Ag 50:50 MPCs is shown in Figure 3.2.

Evaluation of Catalytic Activity of MPCs. Catalytic activity was evaluated by dissolving $\sim 8 \mathrm{mg}$ MPCs in $8 \mathrm{~mL}$ dichloromethane. $400 \mu \mathrm{L}$ of allyl alcohol was added to the reaction vessel and stirred for $10 \mathrm{~min}$. A sample was then taken, representing the $\mathrm{T}_{0}$ sample. $\mathrm{H}_{2}$ was then bubbled at a rate of $8 \mathrm{~mL} / \mathrm{min}$, and an aliquot removed at $15 \mathrm{~min}$ intervals for analysis by GC up to a final time of $120 \mathrm{~min}$. 
The samples were evaluated using an SRI gas chromatograph with a 6' Carbowax 20M 1/8" packed column using on-column injection. A flame ionization detector was used, with an initial temperature of $60{ }^{\circ} \mathrm{C}$ ramped to $120{ }^{\circ} \mathrm{C}$ at a rate of 10 ${ }^{\circ} \mathrm{C} / \mathrm{min}$.

\subsection{RESULTS AND DISCUSSION}

\section{UV-vis Characterization of MPCs}

When dissolved in toluene, pure Pd MPCs show an absorbance peak at around 300 $\mathrm{nm}$ (Figure 3.2A). As can be seen, C12S Pd MPCs show excellent stability over $1 \mathrm{~h}$ of $\mathrm{H}_{2}$ treatment. By contrast, pure Ag MPCs show a pronounced plasmon band with a peak absorbance of around 450nm, although these are also $\mathrm{H}_{2}$ stable (Figure 3.2B).

Interestingly, when C12S 50:50 Pd:Ag Alloy MPCs are prepared by co-reduction, no Ag plasmon band is visible. These MPCs retain excellent stability in $\mathrm{H}_{2}$ (Figure 3.3A). By contrast, C12S 50:50 Pd:Ag MPCs prepared by galvanic exchange show the presence of a Ag plasmon band along with a second plasmon band at 370nm (Figure 3.3B). We have not been able to determine the source of this second plasmon band. The presence of the strong Ag plasmon band suggests that galvanic exchange may not have worked very well in this case.

\section{Galvanic Exchange}

UV-VIS data showed that galvanic exchange of Pd onto the Ag MPCs was at least somewhat successful for the C12S Ag MPCs. There was a change in the Ag UV-vis 

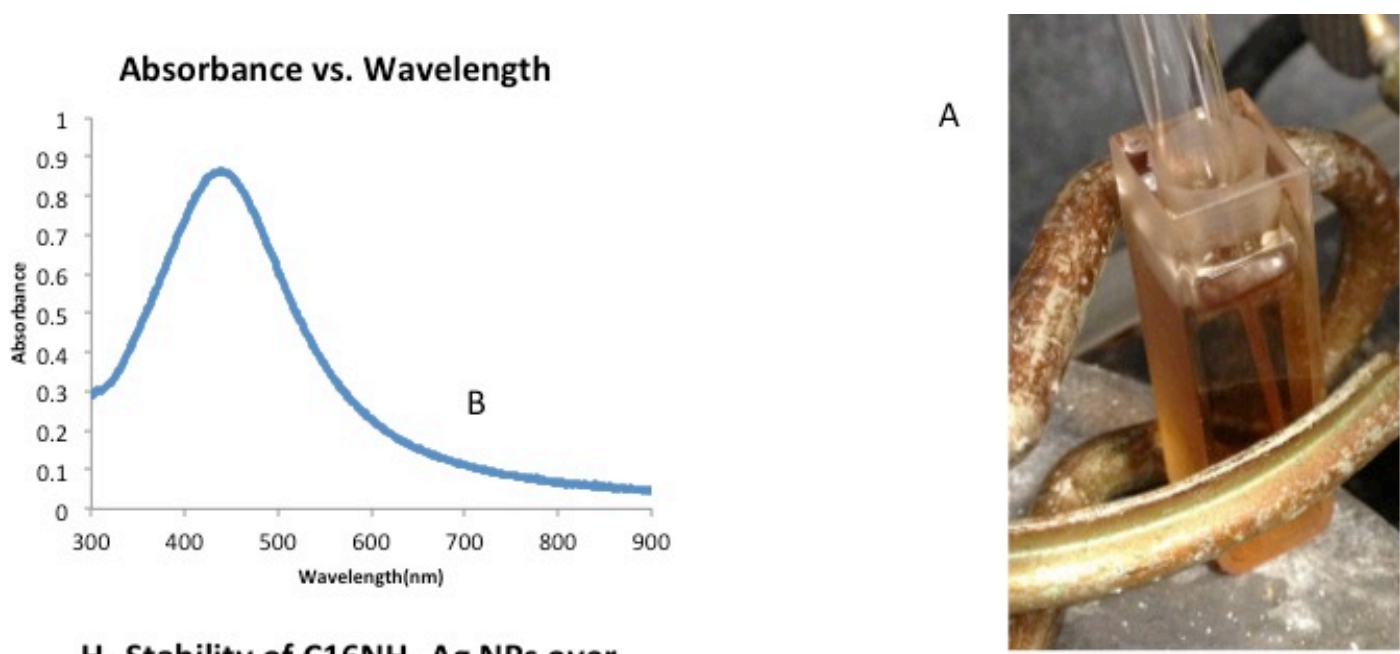

\section{$\mathrm{H}_{2}$ Stability of $\mathrm{C}_{16 \mathrm{NH}_{2}} \mathrm{Ag}$ NPs over \\ Time}

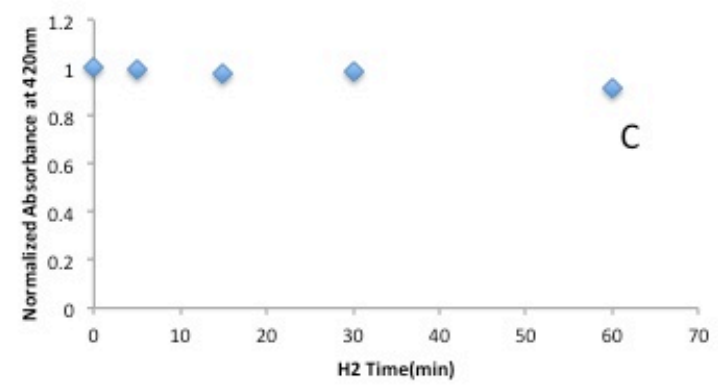

Figure 3.1. (A) Experimental Set-up for examining $\mathrm{H}_{2}$ stability.

(B) Absorbance vs. Wavelength plot for Ag C12S MPCs in solution.

(C) Stability of Ag MPCs based on the absorbance as a function of time from 0-60 min under bubbling $\mathrm{H}_{2}$. 
spectrum and a notable change in the physical characteristics of the MPCs dissolved in solution, with pure Ag NPs showing a pronounced amber color. Pd and PdAg alloy MPCs (whether prepared by co-reduction or galvanic exchange) showed a more brownblack color in solution. The presence of the Ag plasmon band in the spectrum after exchange shows that the reaction may not have proceeded to the full extent possible. This could be due to the $\mathrm{PdCl}_{4}{ }^{2-}$ being in the aqueous phase and C12S Ag MPCs being in the toluene phase. The balanced reaction for the galvanic exchange is:

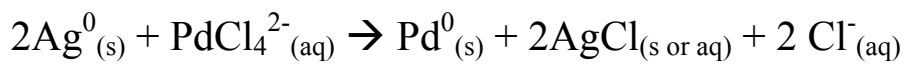

We found it very difficult to synthesize PdAg alloy MPCs with high Pd ratios (greater than 50:50) through a galvanic exchange reaction. As previously discussed, each $\mathrm{Pd}^{0}$ atom replaces two $\mathrm{Ag}^{0}$ atoms in the core of the MPC. The net result of this is a dramatic reduction in the size of the MPCs at higher Pd:Ag ratios. Through the course of this study, it was found to not be possible to isolate these MPCs. Future work may examine these alloy MPCs with higher MPC ratios if isolation is successful.

\section{Catalytic Efficiency}

Initial studies showed that while C12S Pd MPCs were fairly efficient catalysts, catalytic efficiency dropped off dramatically with even $10 \%$ silver as prepared by coreduction. As consistent with the data reported by Moreno, ${ }^{46,47}$ the catalytic turnover frequency (TOF) for C12S Pd MPCs for hydrogenation/isomerization of allyl alcohol was 36. A gas chromatogram following the hydrogenation/isomerization of allyl alcohol using pure Pd C12S MPCs as a catalyst is shown in Figure 3.4. (Frame A) and using C12S Pd:Ag 50:50 NPs prepared by co-reduction (Frame B). With C12S Pd MPCs, there is complete 

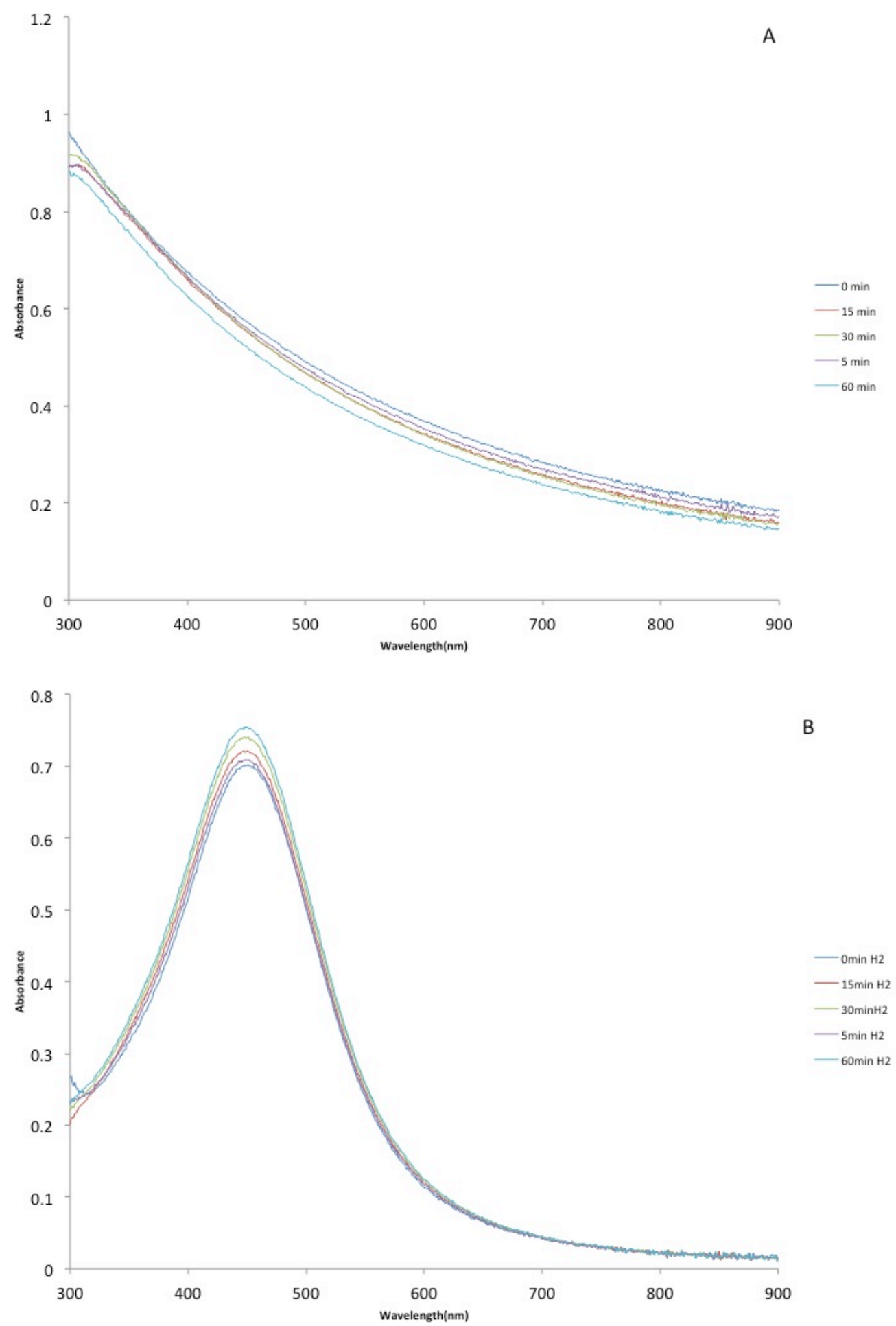

Figure 3.2. (A) $\mathrm{H}_{2}$ Stability of $\mathrm{C} 12 \mathrm{~S}$ Pd MPCs over time. (B) $\mathrm{H}_{2}$ Stability of C12S Ag MPCs over time. 

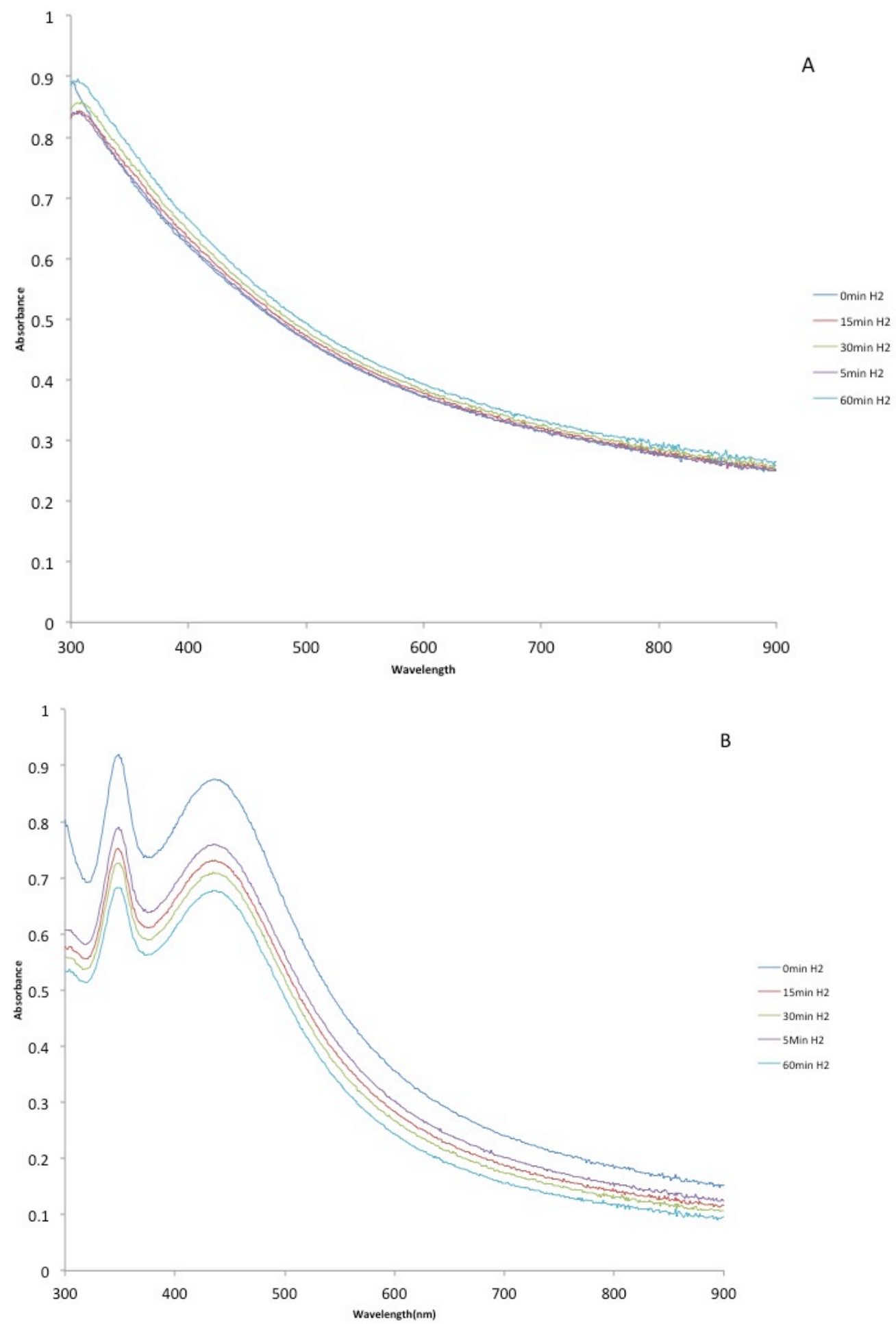

Figure 3.3. UV-Vis spectrum showing $\mathrm{H}_{2}$ stability of $\mathrm{C} 12 \mathrm{~S} \mathrm{Ag}: \mathrm{Pd}$

50:50 MPCs prepared by (A) co-reduction and (B) galvanic exchange. 
conversion of allyl alcohol to the propionaldehyde (isomerization product) as indicated by the labeled peaks in the GC. With the C12S Pd:Ag 50:50 MPCs (co-reduction), there is little evidence of reaction products. We believe the decrease in the allyl alcohol peak is mainly due to evaporation. The catalytic data is also summarized in Figure 3.5 (Frames A and B), where full conversion of allyl alcohol occurred within 30 min with C12S Pd MPCs but no conversion occurred for 60 min with C12S 50:50 Pd:Ag MPCs (coreduction)..

Figure 3.6 shows all of the Pd:Ag ratios studied using co-reduction and galvanic exchange as the synthesis methods. As expected, pure Ag MPCs showed no catalytic activity $(\mathrm{TOF}=0)$. In addition PdAg MPCs with a ratio of 50:50 Pd:Ag ratio showed no catalytic activity regardless of the method of preparation (co-reduction vs. galvanic exchange). Catalysts prepared by co-reduction at relatively high palladium concentrations show very high catalytic activity, but still significantly decreased from pure C12S Pd MPCs. For C12S Pd MPCs, a turnover frequency of 36 was observed. This is consistent with the data reported by Moreno et al. of a TOF of $44 .{ }^{47}$ With the addition of even $10 \% \mathrm{Ag}$ through co-reduction, C12S protected MPCs show a dramatically reduced TOF to 6.2. These alloy MPCs protected by thiol ligands were not studied by Moreno, so no comparison data is available. At a 7:3 Pd:Ag ratio, no catalytic activity was observed in MPCs prepared by co-reduction. This is shown in Figure 3.6.

As previously mentioned, increasingly high Pd content results in decreasing size of the metal core of the MPCs for those prepared by galvanic exchange. Thus, at higher Pd 

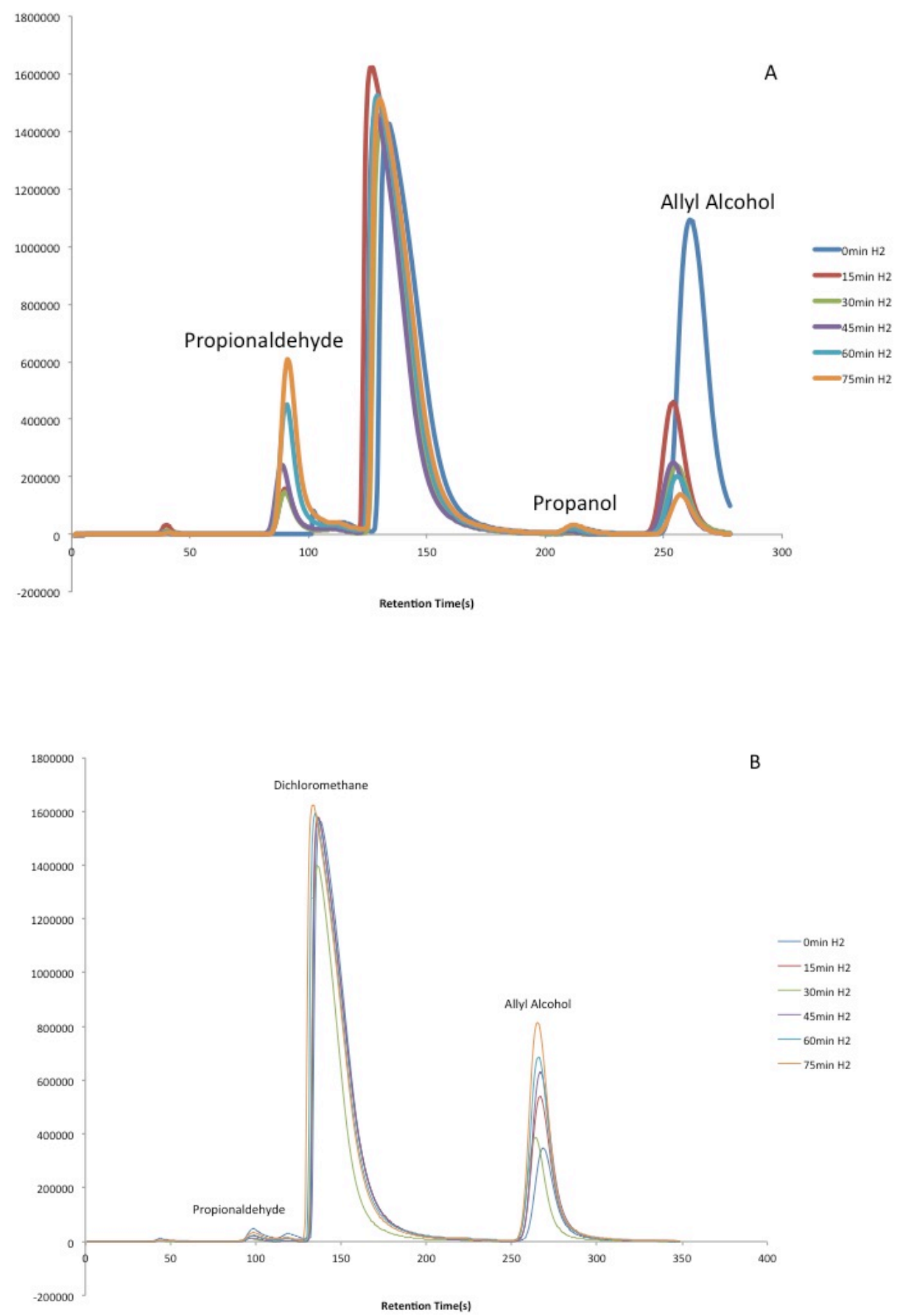

Figure 3.4. Gas chromatograms monitoring the hydrogenation/isomerization of allyl alcohol with $\mathrm{H}_{2}$ and the catalyst (A) C12S Pd MPCs and (B) C12S Pd:Ag 50:50 MPCs prepared by co-reduction. 

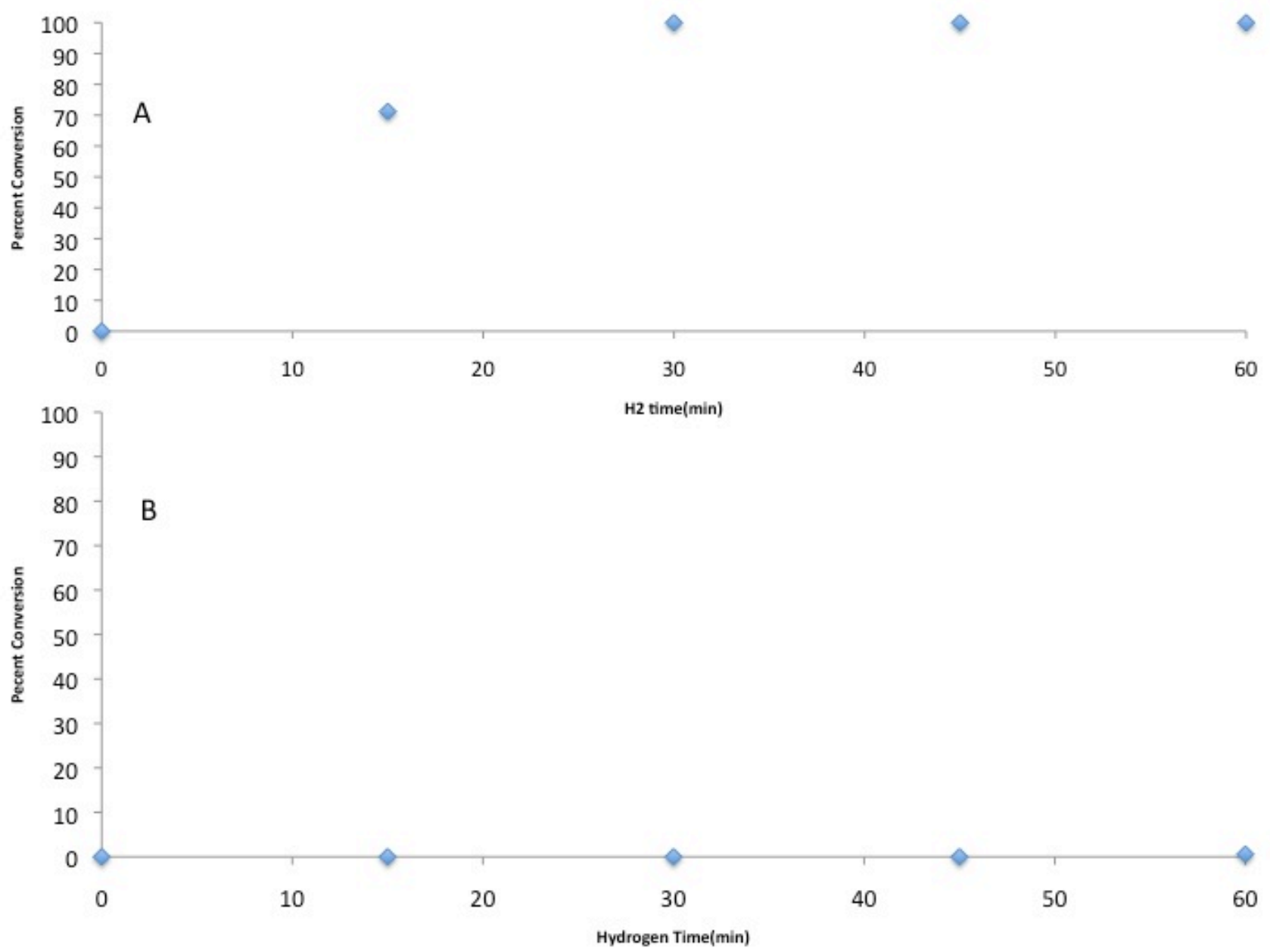

Figure 3.5. (A) Percent conversion of allyl alcohol vs. time plot for (A) pure Pd C12S MPCs and (B) C12S 50:50 Pd:Ag MPCs prepared by co-reduction. 


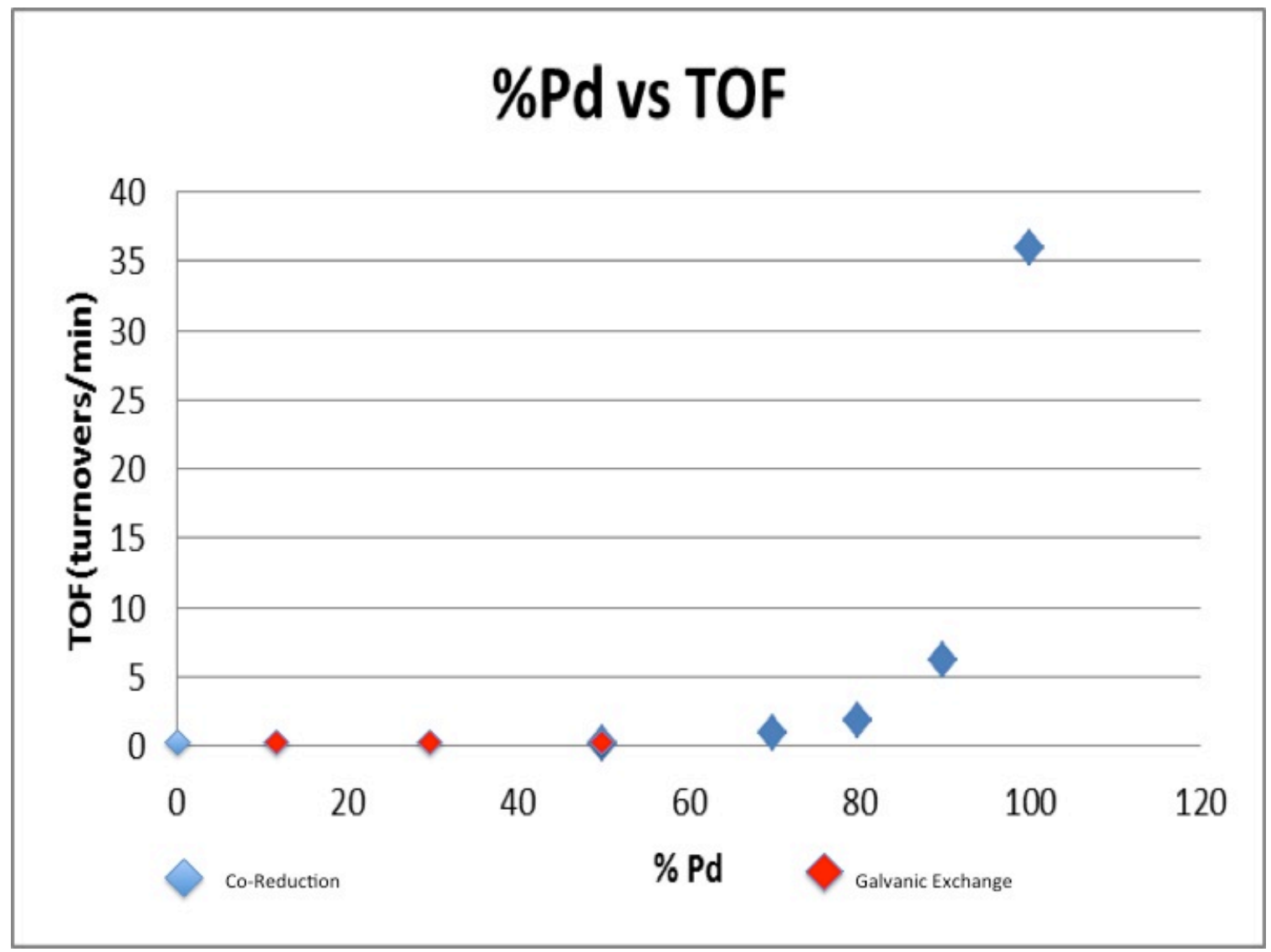

Figure 3.6. Plot illustrating percent conversion of allyl alcohol vs. Pd:Ag ratio for both co-reduction and galvanic exchange synthesis. 
concentrations, MPCs prepared by galvanic exchange were not possible for us to isolate using the techniques described here. It was hoped that MPCs prepared by galvanic exchange would show higher catalytic efficiency with the same amount of Pd prepared by co-reduction. This, however, was not properly tested since we were not able to prepare and isolate any PdAg MPCs with $>50 \%$ Pd by galvanic exchange. Catalytic performance, as measured in terms of TOF, was equally poor with MPCs prepared by galvanic exchange up to $50 \% \mathrm{Pd}$, however (Figure 3.7).

\section{Synthesis of Ag MPCs in the presence of $\mathrm{C}_{16 \mathrm{NH}_{2}}$}

Ag MPCs synthesized in the presence of $\mathrm{C} 16 \mathrm{NH}_{2}$ stabilizer were successfully prepared using methods previously described for amine-coated MPCs in Chapter 2. It was also found that a successful galvanic exchange could not be carried out.

\subsection{CONCLUSIONS}

\section{Catalytic efficiency of C12S PdAg MPCs prepared by co-reduction}

As was shown in the data, the catalytic efficiency rapidly decreased with the addition of relatively small amounts of $\mathrm{Ag}$ to the metal core. Moreno ${ }^{47}$ reported relatively high TOFs with $\mathrm{C} 16 \mathrm{NH} 2$-coated (likely a nitrile actually) MPCs with alloy cores consisting of $10 \% \mathrm{Ag}$, but this was not the case for those stabilized with $\mathrm{C} 12 \mathrm{~S}$. The TOF dropped by a factor of about 6 with $10 \% \mathrm{Ag}$. 
50:50 Pd:Ag C12S By Galvanic Exchange

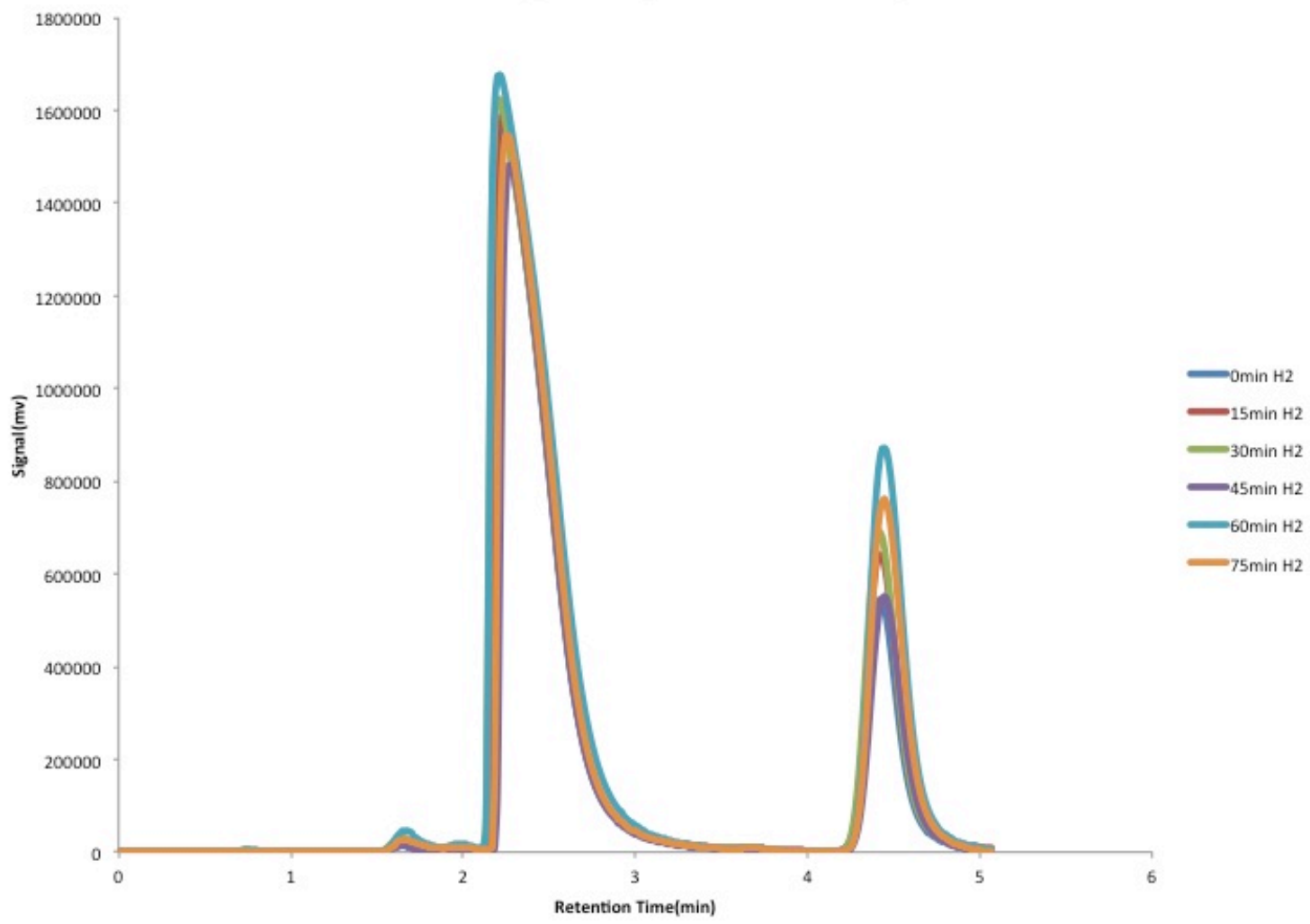

Figure 3.7. Combined 0-75min Chromatograms for 50:50 Pd:Ag C12S MPCs prepared by galvanic exchange. 
As discussed, however, it was not possible to achieve galvanic exchange of such alkylamine coated MPCs, so a direct comparison with co-reduced $\mathrm{C}_{16 \mathrm{NH}_{2}} \mathrm{PdAg} \mathrm{MPCs}$ was not possible.

\section{Catalysis with C12S PdAg MPCs prepared by galvanic exchange}

As would be expected, pure Ag MPCs showed no catalytic activity for hydrogenation or isomerization of allyl alcohol. Similarly, C12S PdAg MPCs with 50\% Pd prepared by galvanic exchange also showed no catalytic activity. As discussed, we were not able to isolate C12S AgPd MPCs with Pd content higher than 50\% when synthesized by galvanic exchange, which prevented any conclusion to be drawn with regard to the potential catalytic activity for those $>50 \% \mathrm{Pd}$ by galvanic exchange. In

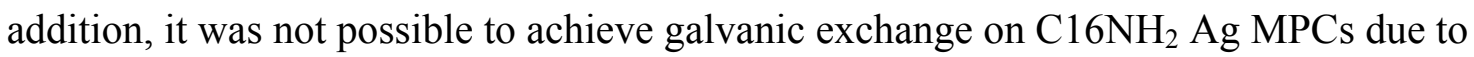
stability issues. If a technique could be found to achieve this exchange, such MPCs might hold promise for catalytic applications.

From the results presented herein though, we currently conclude that PdAg alloy MPCs show little promise for catalytic applications. If the problem of isolation of particles with high Pd ratios could be solved, as well as successful galvanic exchange of alkylamine (nitrile)-coated MPCs, this strategy might show promise for designing less expensive, active catalysts in the future. 


\section{CHAPTER 4}

\section{CONCLUSIONS AND FUTURE DIRECTIONS}

\subsection{Study of Pd MPCs Synthesized in the Presence of Octylamine}

The data from this study conclusively shows the amine functional group is converted to a nitrile group during attachment to Pd MPCs. The Pd obviously plays a functional role in this conversion, as the same conversion does not occur when Au MPCs are synthesized in the presence of amines.

Furthermore, when the ligands are removed by $\mathrm{H}_{2}$ or ligand place exchange, some conversion of the nitrile ligand to the acid and amide functional groups occurs. Dissolved $\mathrm{O}_{2}$ plays a role in this conversion, as the conversion does not occur when the reaction is performed under an inert $\left(\mathrm{N}_{2}\right)$ atmosphere. The only product seen results from simple desorption of the nitrile ligands from the surface of the $\mathrm{Pd}$, with no conversion from nitrile to acid or amide.

Interestingly, the conversion also does not occur when the ligands are removed from the MPCs by heating, even when this heating occurs under ambient conditions. Apparently, heating only promotes simple desorption of the ligands from the Pd MPCs, showing that an $\mathrm{H}$ source is needed for conversion to amine or acid products.

\subsection{Future Directions of C7CN Pd MPC Studies}

The amide to nitrile conversion which occurs during Pd MPC synthesis is interesting and could provide for interesting organic synthesis applications. Future studies may look at scaling up this reaction for use on large-scale organic synthesis reactions. 
In addition, the conversion of nitrile ligands to amides and acids with $\mathrm{C} 7 \mathrm{CN}$ coated Pd MPCs could further be used in organic synthesis applications. It would be interesting to examine if adding additional $\mathrm{O}_{2}$ (such as by bubbling along with $\mathrm{H}_{2}$ ) to the solution could result in more complete conversion of the nitrile ligand into the amine and acid products, although achieving some selectivity in this reaction would also be desirable from an organic synthesis standpoint.

Finally, it would be interesting to examine if Pd MPCs can be used to convert excess nitrile in the solution to the amine and acid products. If excess nitrile in the solution could result in continuous turn-over of nitrile to amine and acid products, this could again be potentially used for synthetic applications.

\subsection{Conclusions from PdAg Alloy MPCs}

As shown in the data presented herein, the Pd C12S catalyst is effective in the allyl alcohol isomerization reaction. Adding even a small amount of $\mathrm{Ag}$ to the palladium core $(10 \%)$ dramatically decreases the catalytic activity as measured in terms of TOF. It was hoped that by preparing these PdAg alloy MPCs through galvanic exchange, there would be more Pd atoms on the surface that would allow them to be more effective catalysts, even with large amounts of Ag in the alloy. Unfortunately, this hypothesis could not be tested because of our inability to isolate stable PdAg alloy MPCs with high Pd content by galvanic exchange. 


\subsection{Future Directions of PdAg Alloy MPC Catalysts}

Moreno et al., ${ }^{47}$ demonstrated efficacy of PdAg alloy catalysts prepared by coreduction using $\mathrm{C}_{16 \mathrm{NH}_{2}}$ stabilizing ligands(or more correctly $\mathrm{C} 15 \mathrm{CN}$ ) when relatively small amounts of $\mathrm{Ag}(\sim 10 \%)$ were present. Moreno also demonstrated that as a general trend these were more active catalysts because of their greater stability through the course of the reaction. If the same MPCs were prepared by galvanic exchange, it is possible that such MPCs might show even better catalytic activity. A successful synthesis strategy should be explored more in the future. PdAg MPCs could also be tested that were not purified to get an idea of their potential as catalysts. More work should also be performed to isolate the various C12S Pd and PdAg MPCs by the two synthesis methods in order to prove the concept that the method of synthesis can dramatically effect catalytic function, even though the $\mathrm{C} 12 \mathrm{~S}$ is not the best ligand in terms of achieving the highest TOFs. A third strategy could be performing chemical reduction of Pd(II) directly on Ag MPCs as a different method to make core/shell Ag/Pd MPCs. 


\section{REFERENCES}

(1) Bonnemann, H.; Richards, R. M. Eur. J. Inorg. Chem. 2001, 2455.

(2) Alkilany, A. M.; Murphy, C. J. Langmuir 2009, 25, 13874.

(3) Chen, Y.; Luo, X.; She, H.; Yue, G.-H.; Peng, D.-L. J Nanosci Nanotechnol 2009, 9, 5157.

(4) Kievit, F. M.; Zhang, M. Acc. Chem. Res. 2011, 44, 853.

(5) Castro, E. G.; Salvatierra, R. V.; Schreiner, W. H.; Oliveira, M. M.; Zarbin, A. J. G. Chem. Mater. 2010, 22, 360.

(6) Nakhaei, P. A.; Housaindokht, M. Catal. Lett., Ahead of Print.

(7) Khamdokhov, A. Z.; Teshev, R. S.; Khamdokhov, Z. M.; Kulikauskas, V. S.; Chernykh, P. N. J. Surf. Invest.: X-Ray, Synchrotron Neutron Tech. 2013, 7, 737.

(8) Kondratenko, E. V.; Amrute, A. P.; Pohl, M.-M.; Steinfeldt, N.; Mondelli, C.; Perez-Ramirez, J. Catal. Sci. Technol. 2013, 3, 2555.

(9) Zapf, R.; Thiele, R.; Wichert, M.; O'Connell, M.; Ziogas, A.; Kolb, G. Catal. Commun. 2013, 41, 140.

(10) Low, J. E.; Foelske-Schmitz, A.; Krumeich, F.; Woerle, M.; Baudouin, D.; Rascon, F.; Coperet, C. Dalton Trans. 2013, 42, 12620.

(11) Calver, C. F.; Dash, P.; Scott, R. W. J. ChemCatChem 2011, 3, 695.

(12) Garcia-Martinez, J. C.; Lezutekong, R.; Crooks, R. M. J. Am. Chem. Soc. 2005, 127, 5097.

(13) Chen, X.; Wang, H.; He, J.; Cao, Y.; Cui, Z.; Liang, M. J. Nanosci. Nanotechnol. 2010, 10, 3138.

(14) Liu, Y.; Liu, B.; Liu, Y.; Wang, Q.; Hu, W.; Jing, P.; Liu, L.; Yu, S.; Zhang, J. Appl. Catal., B 2013, 142-143, 615.

(15) Shaabani, A.; Mahyari, M. Catal. Lett., Ahead of Print.

(16) Moreno, M.; Kissell, L. N.; Jasinski, J. B.; Zamborini, F. P. ACS Catal. 2012, 2, 2602.

(17) Gary-Bobo, M.; Vaillant, O.; Maynadier, M.; Basile, I.; Gallud, A.; El, C. K.; Bouffard, E.; Morere, A.; Rebillard, X.; Puche, P.; Nirde, P.; Garcia, M. Curr. Med. Chem. 2013, 20, 1946.

(18) Rieter, W. J.; Pott, K. M.; Taylor, K. M. L.; Lin, W. J. Am. Chem. Soc. 2008, $130,11584$.

(19) Zhao, Z.; Sevryugina, Y.; Carpenter, M. A.; Welch, D.; Xia, H. Anal. Chem. 2004, 76, 6321.

(20) Moreno, M.; Ibanez, F. J.; Jasinski, J. B.; Zamborini, F. P. J. Am. Chem. Soc. 2011, 133, 4389.

7852.

(21) Astruc, D.; Lu, F.; Aranzaes, J. R. Angew Chem Int Ed Engl 2005, 44,

(22) Narayanan, R.; El-Sayed, M. A. J. Am. Chem. Soc. 2003, 125, 8340.

(23) Mandal, S.; Roy, D.; Chaudhari, R. V.; Sastry, M. Chem. Mater. 2004, 16, 3714. 
(24) Wilson, O. M.; Knecht, M. R.; Garcia-Martinez, J. C.; Crooks, R. M. J. Am. Chem. Soc. 2006, 128, 4510.

(25) Crooks, R. M.; Zhao, M.; Sun, L.; Chechik, V.; Yeung, L. K. Acc. Chem. Res. 2001, 34, 181.

(26) Sadeghmoghaddam, E.; Lam, C.; Choi, D.; Shon, Y.-S. J. Mater. Chem. 2011, 21, 307.

(27) Zamborini, F. P.; Smart, L. E.; Leopold, M. C.; Murray, R. W. Anal. Chim. Acta 2003, 496, 3 .

(28) Beeram, S. R.; Zamborini, F. P. J. Am. Chem. Soc. 2009, 131, 11689.

(29) Abdelmoti, L. G.; Zamborini, F. P. Langmuir 2010, 26, 13511.

(30) Beeram, S. R.; Zamborini, F. P. ACS Nano 2010, 4, 3633.

(31) Beeram, S. R.; Zamborini, F. P. J. Phys. Chem. C 2011, 115, 7364.

(32) Chauhan, R.; Moreno, M.; Banda, D. M.; Zamborini, F. P.; Grapperhaus, C. A. RSC $A d v$. 2014, 4, 46787.

(33) Ivanova, O. S.; Zamborini, F. P. Anal. Chem. (Washington, DC, U. S.) 2010, 82,5844 .

(34) Masitas, R. A.; Zamborini, F. P. J. Am. Chem. Soc. 2012, 134, 5014.

(35) Fang, A.; White, S.; Jain, P. K.; Zamborini, F. P. Nano Lett. 2015, 15, 542.

(36) Slawinski, G. W.; Zamborini, F. P. Langmuir 2007, 23, 10357.

(37) Ibanez, F. J.; Zamborini, F. P. ACS Nano 2008, 2, 1543.

(38) Ivanova, O. S.; Zamborini, F. P. J. Am. Chem. Soc. 2010, 132, 70.

(39) Zamborini, F. P.; Gross, S. M.; Murray, R. W. Langmuir 2001, 17, 481.

(40) Ibanez, F. J.; Zamborini, F. P. Langmuir 2006, 22, 9789.

(41) Dasari, R.; Zamborini, F. P. J. Am. Chem. Soc. 2008, 130, 16138.

(42) Moreno, M.; Ibanez, F. J.; Jasinski, J. B.; Zamborini, F. P. J. Am. Chem.

Soc. 2011, 133, 4389.

(43) Ibanez, F. J.; Zamborini, F. P. J. Am. Chem. Soc. 2008, 130, 622.

(44) Slawinski, G. W.; Ivanova, O. S.; Zamborini, F. P. Langmuir 2011, 27 , 13293.

(45) Ibanez, F. J.; Zamborini, F. P. Small 2012, 8, 174.

(46) Moreno, M.; Kissell, L. N.; Jasinski, J. B.; Zamborini, F. P. ACS Catal. 2012, 2, 2602.

(47) Moreno, M., University of Louisville, 2013.

(48) Brust, M.; Walker, M.; Bethell, D.; Schiffrin, D. J.; Whyman, R. J. Chem. Soc., Chem. Commun. 1994, 801.

(49) Mieszawska, A. J.; Jalilian, R.; Sumanasekera, G. U.; Zamborini, F. P. J. Am. Chem. Soc. 2005, 127, 10822.

(50) Halada, G. P.; Jha, P. K.; Cuiffo, M. A.; The Research Foundation of State University of New York, USA . 2013, p 21pp.

(51) Rabinal, M. K.; Kalasad, M. N.; Praveenkumar, K.; Bharadi, V. R.; Bhikshavartimath, A. M. J. Alloys Compd. 2013, 562, 43.

(52) Richter, K.; Birkner, A.; Mudring, A.-V. Angew. Chem., Int. Ed. 2010, 49, 2431.

(53) Brust, M.; Walker, M.; Bethell, D.; Schiffrin, D. J.; Whyman, R. J. Chem. Soc., Chem. Commun. 1994, 801. 
(54) Duran, N.; Marcato, P. D.; Alves, O. L.; Souza, G. I. H. D.; Esposito, E. J Nanobiotechnology 2005, 3, 8 .

(55) Richter, K.; Campbell, P. S.; Baecker, T.; Schimitzek, A.; Yaprak, D.; Mudring, A.-V. Phys. Status Solidi B 2013, 250, 1152.

(56) Kowalska, E.; Czerwosz, E.; Diduszko, R.; Kaminska, A.; Danila, M. Sens. Actuators, A, Ahead of Print.

(57) Cadierno, V.; Garcia-Garrido, S. E.; Gimeno, J.; Varela-Alvarez, A.; Sordo, J. A. J. Am. Chem. Soc. 2006, 128, 1360.

(58) Yamauchi, M.; Kobayashi, H.; Kitagawa, H. ChemPhysChem 2009, 10, 2566.

(59) Horinouchi, S.; Yamanoi, Y.; Yonezawa, T.; Mouri, T.; Nishihara, H. Langmuir 2006, 22, 1880.

(60) Ibanez, F. J.; Zamborini, F. P. J. Am. Chem. Soc. 2008, 130, 622.

(61) Li, Y.; El-Sayed, M. A. J. Phys. Chem. B 2001, 105, 8938.

(62) Yeung, L. K.; Crooks, R. M. Nano Lett. 2001, 1, 14.

(63) Chen, S.; Ostrom, C.; Chen, A. Int. J. Hydrogen Energy, Ahead of Print.

(64) Rubio-Marques, P.; Leyva-Perez, A.; Corma, A. Chem. Commun.

(Cambridge, U. K.) 2013, 49, 8160.

(65) Iranpoor, N.; Firouzabadi, H.; Rostami, A. Appl. Organomet. Chem. 2013, 27,501 .

(66) Liang, Q.; Liu, J.; Wei, Y.; Zhao, Z.; MacLachlan, M. J. Chem. Commun. (Cambridge, U. K.) 2013, 49, 8928.

(67) Zhao, Y.; Jia, L.; Medrano, J. A.; Ross, J. R. H.; Lefferts, L. ACS Catal., Ahead of Print.

(68) Samarasimhareddy, M.; Prabhu, G.; Vishwanatha, T. M.; Sureshbabu, V. V. Synthesis 2013, 45, 1201.

(69) Rasouli, M. A.; Ranjbar, P. R. Z. Naturforsch., B. J. Chem. Sci. 2013, 68,

946.

(70) Cui, Z.-M.; Mechai, A.; Guo, L.; Song, W.-G. RSC Adv. 2013, 3, 14979.

(71) Gomez, M. V.; Guerra, J.; Velders, A. H.; Crooks, R. M. J. Am. Chem. Soc. 2009, 131, 15564.

(72) Andres, R.; De, J. E.; Flores, J. C. New J. Chem. 2007, 31, 1161.

(73) Yamamoto, Y.; Miyachi, M.; Yamanoi, Y.; Minoda, A.; Maekawa, S.; Oshima, S.; Kobori, Y.; Nishihara, H. J. Inorg. Organomet. Polym. Mater., Ahead of Print.

(74) Wuelfing, W. P.; Zamborini, F. P.; Templeton, A. C.; Wen, X.; Yoon, H.; Murray, R. W. Chem. Mater. 2001, 13, 87.

(75) Ibanez, F. J.; Zamborini, F. P. Langmuir 2006, 22, 9789.

(76) Sen, F.; Goekagac, G. J. Phys. Chem. C 2007, 111, 1467.

(77) Zamborini, F. P.; Gross, S. M.; Murray, R. W. Langmuir 2001, 17, 481.

(78) Chiu, S.-Y.; Huang, H.-W.; Huang, T.-H.; Liang, K.-C.; Liu, K.-P.; Tsai, J.-H.; Lour, W.-S. Sens. Actuators, B 2009, 138, 422.

(79) RaviPrakash, J.; McDaniel, A. H.; Horn, M.; Pilione, L.; Sunal, P.; Messier, R.; McGrath, R. T.; Schweighardt, F. K. Sens. Actuators, B 2007, 120, 439.

(80) Zamborini, F. P.; Crooks, R. M. Langmuir 1997, 13, 122. 
(81) Hostetler, M. J.; Wingate, J. E.; Zhong, C.-J.; Harris, J. E.; Vachet, R. W.; Clark, M. R.; Londono, J. D.; Green, S. J.; Stokes, J. J.; Wignall, G. D.; Glish, G. L.;

Porter, M. D.; Evans, N. D.; Murray, R. W. Langmuir 1998, 14, 17.

(82) Zamborini, F. P.; Campbell, J. K.; Crooks, R. M. Langmuir 1998, 14, 640.

(83) Zamborini, F. P.; Crooks, R. M. Langmuir 1998, 14, 3279.

(84) Hostetler, M. J.; Templeton, A. C.; Murray, R. W. Langmuir 1999, 15, 3782.

(85) Cliffel, D. E.; Zamborini, F. P.; Gross, S. M.; Murray, R. W. Langmuir 2000, 16, 9699 .

(86) Templeton, A. C.; Zamborini, F. P.; Wuelfing, W. P.; Murray, R. W. Langmuir 2000, 16, 6682.

(87) Zamborini, F. P.; Leopold, M. C.; Hicks, J. F.; Kulesza, P. J.; Malik, M. A.; Murray, R. W. J. Am. Chem. Soc. 2002, 124, 8958.

(88) Sadeghmoghaddam, E.; Lam, C.; Choi, D.; Shon, Y.-S. J. Mater. Chem. 2011, 21, 307.

(89) Imada, Y.; Ohsaki, M.; Noguchi, M.; Maeda, T.; Fujiki, M.; Kawamorita, S.; Komiya, N.; Naota, T. ChemCatChem 2015, 7, 99.

(90) Wisniewski, W.; Nikczynski, G.; Scigalski, F. Chemik 2011, 65, 261.

(91) Zaramella, D.; Scrimin, P.; Prins, L. J. J. Am. Chem. Soc. 2012, 134, 8396.

(92) Lee, M.; Amaratunga, P.; Kim, J.; Lee, D. J. Phys. Chem. C 2010, 114, 18366.

(93) Bonomi, R.; Selvestrel, F.; Lombardo, V.; Sissi, C.; Polizzi, S.; Mancin, F.; Tonellato, U.; Scrimin, P. J. Am. Chem. Soc. 2008, 130, 15744.

(94) Kesavan, L.; Tiruvalam, R.; Ab Rahim, M. H.; bin Saiman, M. I.; Enache, D. I.; Jenkins, R. L.; Dimitratos, N.; Lopez-Sanchez, J. A.; Taylor, S. H.; Knight, D. W.; Kiely, C. J.; Hutchings, G. J. Science (Washington, DC, U. S.) 2011, 331, 195.

(95) Shon, Y.-S.; Dawson, G. B.; Porter, M.; Murray, R. W. Langmuir 2002, 18,3880 .

(96) Calver, C. F.; Dash, P.; Scott, R. W. J. ChemCatChem 2011, 3, 695.

(97) Li, Z.-P.; Gao, J.; Xing, X.-T.; Wu, S.-Z.; Shuang, S.-M.; Dong, C.; Paau, M.-C.; Choi, M. M. F. J. Phys. Chem. C 2010, 114, 723.

(98) Newman, J. D. S.; Blanchard, G. J. Langmuir 2006, 22, 5882.

(99) Delcamp, J. H.; Gormisky, P. E.; White, M. C. J. Am. Chem. Soc. 2013, 135,8460 .

(100) Shimizu, K.-i.; Imaiida, N.; Sawabe, K.; Satsuma, A. Appl. Catal., A 2012, 421-422, 114.

(101) Zhao, Z.; Sevryugina, Y.; Carpenter, M. A.; Welch, D.; Xia, H. Anal. Chem. 2004, 76, 6321. 
University of Louisville Department of Chemistry 2320 S. Broook St

Louisville, KY 40292

\section{CURRICULUM VITAE / XMKH]\%HQNP IQ+ XWKHURQ}

lbhutc01@louisville.edu 502-330-2132

Current as of April 2014

\section{Education}

University of Louisville, Anticipated Graduation May 2015, Masters of Science

- Degree completed under the direction of Prof. Francis P. Zamborini

- Acquired experience in synthesizing a variety of nanoparticles, including palladium, silver, gold, and alloys of those

- Acquired experience in characterization of those nanoparticles

- Served as a graduate teaching assistant for 4 years

- Mentored undergraduate student in synthesis, characterization, and catalysis of nanoparticles

Georgetown College, B.S. in Chemistry, May 2010

- Completed a minor in physics

- Served as a teaching assistant in the chemistry department for both general chemistry and organic chemistry laboratory courses for 3 years

- Operated, maintained, and repaired department instruments

- Worked two summers as a teaching assistant for PAEMS, a math and science summer camp for high school students.

- Was awarded a GC-PALS fellowship, funded by the Howard Hughes Medical Institute, for summer research

\section{Teaching Experience}

- Graduate Teaching Assistant at University of Louisville, 2011-2015

- Teaching assistant at Georgetown College, 2007-2010

\section{Courses taught as Teaching Assistant}

- General Chemistry laboratory

- Organic Chemistry Laboratory

- Chemistry 105 , an intensive course combining general, organic, and biochemistry for pre-health majors 


\section{Publications}

Exploring the Ligand Chemistry of Pd Nanoparticles Synthesized in the Presence of Octylamine Before and After Exposure to Hydrogen, Heat, and Thiol Place-Exchange Reactions, Luther B. Hutcherson, Monica Moreno and Francis P. Zamborini. Preparing for submission

\section{Conference Presentations}

Investigation of ligand removal from octyl amine coated palladium nanoparticles, Luther B. Hutcherson and Francis P. Zamborini. $246^{\text {th }}$ ACS National Meeting, Indianapolis, IN, September 2013

Isoindolinone inhibitors of MDM2-p53 through arylation/selective oxidation of Nalkylhydroxylactams, Richard K. Dempster, Luther B. Hutcherson, Frederick A. Luzzio. $42^{\text {nd }}$ Central Regional Meeting of the American Chemical Society, Indianapolis, IN, June 2012 